\title{
Joint Discussion 17 \\ Highlights of recent progress in the seismology of the Sun and Sun-like stars
}

\author{
Timothy R. Bedding ${ }^{1}$, Allan S. Brun ${ }^{2}$,
} Jørgen Christensen-Dalsgaard ${ }^{3}$, Ashley Crouch ${ }^{4}$, Peter De Cat ${ }^{5}$, Raphael A. García ${ }^{2}$, Laurent Gizon ${ }^{6}$, Frank Hill ${ }^{7}$, Hans Kjeldsen ${ }^{3}$, John W. Leibacher ${ }^{7,8,9}$ (ed.), Jean-Pierre Maillard ${ }^{10}$, S. Mathis ${ }^{5}$, M. Cristina Rabello-Soares ${ }^{11}$, Jean-Pierre Rozelot ${ }^{12}$, Matthias Rempel ${ }^{13}$, Ian W. Roxburgh ${ }^{14}$, Réza Samadi ${ }^{9}$, Suzanne Talon ${ }^{15}$, and Michael J. Thompson ${ }^{16}$

${ }^{1}$ School of Physics, University of Sydney, NSW 2006, Australia email: bedding@physics.usyd.edu.au

${ }^{2}$ DSM/DAPNIA/Service d'Astrophysique \& UMR AIM 7158,

CEA Saclay, F-91191 Gif-sur-Yvette, France email: sacha.brun,rafael.garcia,stephane.mathis@cea.fr

${ }^{3}$ Institut for Fysik og Astronomi, Aarhus Universitet, DK-8000 Aarhus C, Denmark email: jcd,hans@phys.au.dk

${ }^{4}$ Canadian Space Agency, Longueuil, QC, J3Y 8Y9, Canada email: ash@astro.umontreal.ca

${ }^{5}$ Royal Observatory Belgium, Avenue Circulaire 3, B-1180 Brussels, Belgium email: peter@oma.be

${ }^{6}$ Max-Planck-Institut für Sonnensystemforschung, D-37191 Katlenburg-Lindau, Germany email: gizon@linmpi.mpg.de

${ }^{7}$ National Solar Observatory, NOAO, 950 N Cherry Ave, Tucson, AZ 85719-4933, USA email: fhill,jleibacher@nso.edu

${ }^{8}$ Institut d'Astrophysique Spatiale, Centre Universitaire d'Orsay, F-91405 Orsay, France

${ }^{9}$ LESIA, Observatoire de Paris, CNRS UMR 8109, F-92195 Meudon, France email: reza.samadi@obspm.fr

${ }^{10}$ Institut d'Astrophysique de Paris, 98bis Bd Arago, F-75014 Paris, France email: maillard@iap.fr

${ }^{11}$ Hansen Experimental Physics Laboratory, Stanford University, CA 94305, USA email: csoares@sun.stanford.edu

${ }^{12}$ Observatoire de la Côte d'Azur, GEMINI, F-06130Grasse, France email: jean-pierre.rozelot@obs-azur.fr

${ }^{13}$ High Altitude Observatory, NCAR, PO Box 3000, Boulder, CO 80307-3000, USA email: rempel@hao.ucar.edu

${ }^{14}$ Astronomy Unit, Queen Mary, University of London, London E1 4NS, UK email: i.w.roxburgh@qmul.ac.uk

${ }^{15}$ Département de Physique, Université de Montréal, QC H3C 3J7, Canada email: talon@astro.umontreal.ca

${ }^{16}$ SP2RC, Department of Applied Mathematics, University of Sheffield, Sheffield S3 7RH, UK email: michael.thompson@sheffield.ac.uk

Abstract. The seismology and physics of localized structures beneath the surface of the Sun takes on a special significance with the completion in 2006 of a solar cycle of observations by the ground-based Global Oscillation Network Group (GONG) and by the instruments on 
board the Solar and Heliospheric Observatory (SOHO). Of course, the spatially unresolved Birmingham Solar Oscillation Network (BiSON) has been observing for even longer. At the same time, the testing of models of stellar structure moves into high gear with the extension of deep probes from the Sun to other solar-like stars and other multi-mode pulsators, with ever-improving observations made from the ground, the success of the MOST satellite, and the recently launched CoRoT satellite. Here we report the current state of the two closely related and rapidly developing fields of helio- and asteroseimology.

Keywords. Sun: interior, Sun: helioseismology, Sun: rotation, stars: interiors, stars: oscillations (including pulsations), stars: rotation, space vehicles: instruments

\section{Global helioseismology and models}

Global helioseismology, with the study of global modes of oscillation of the Sun, has existed for some thirty years. It continues to produce new and challenging results. Of particular current interest are the temporal variations of the modes and the possible detection of gravity $(g)$ modes, both reviewed here. An aspect that still requires improvement is the accurate determination of the mode parameters used in helioseismology: efforts for the high-degree modes are described below. Three-dimensional modelling of the solar internal dynamics are making substantial strides, as A.S. Brun described at the meeting; and as J. Toomre described, there is a fruitful interplay between the observational results of helioseismology and the results from the numerical simulations. Another aspect of state-of-the-art modelling addresses mixing in the Sun by, for example, gravity waves. Such efforts may provide at least part of the answer to why new abundance measurements, which were described by M. Asplund, make agreement between helioseismology and standard solar models worse rather than better. Dynamo modelling is also gaining inspiration and guidance from helioseismic results, as described further below.

Solar Cycle 23 is the first activity cycle to be completely and continuously covered by modern imaging helioseismology observations, in particular those from the Global Oscillation Network Group (GONG) program, and the Michelson-Doppler Imager (MDI) on the Solar and Heliospheric Observatory (SOHO) spacecraft. In addition, the integratedlight Birmingham Solar Oscillation Network (BiSON) has been continuously operational since 1992. This unprecedented coverage allows us to study a number of aspects of the relationship between the oscillation parameters and the activity, as well as changes in the global properties of the dynamics of the solar rotation and convection zone.

Solar oscillations are observed typically either through Doppler velocity shifts of lines formed in the lower atmosphere of the Sun, or through the associated intensity variations. The oscillations are set up by waves that are turbulently excited by subphotospheric convection. If these waves survive long enough as they propagate inside the Sun, they set up resonant global modes. These modes are the objects of study of global helioseismology. Because the Sun is nearly spherical, the horizontal structure of each mode is described by a spherical harmonic of some degree $(\ell)$ and some azimuthal order $(m)$. Being three dimensional, the modes also have structure in the radial direction, which can be labelled by a third integer, the radial order $(n)$. Thus each mode, and its particular frequency $(\omega)$, can be labelled by the three integers $(n, \ell, m)$.

\subsection{Temporal variation of mode parameters}

It has been known for more than two decades that the oscillation frequencies change with the activity cycle. Using ACRIM data, Woodard \& Noyes (1985) found that the frequencies increase as the number of sunspots rises. The shift was seen to increase with frequency, reaching a value of $+0.4 \mu \mathrm{Hz}$ at about $4 \mathrm{mHz}$. Further work showed that the 
shift is highly correlated with various solar activity indices, in particular the global surface magnetic field. Later observations (e.g., Libbrecht \& Woodard 1990) extended the results to higher frequencies and showed that the shift has a maximum of $+0.5 \mu \mathrm{Hz}$ at the photospheric acoustic cutoff value of $5.6 \mathrm{mHz}$, then changes sign and rapidly drops to $-2 \mu \mathrm{Hz}$ within $0.2 \mathrm{mHz}$ of the cutoff. Modern observations with large-scale ring diagrams (How et al. 2006a) have reached even higher frequencies and reveal an even more complicated structure - the shift has a local minimum of $\sim 4 \mu \mathrm{Hz}$ at $6 \mathrm{mHz}$ and then sharply decreases in absolute magnitude once again. These observations suggest different mechanisms for the interaction between the surface magnetic field, the trapped subsurface $p$ modes below $5.6 \mathrm{mHz}$, and the higher-frequency propagating atmospheric waves. The $p$ modes sense magnetic changes to the acoustic cutoff frequency, while the atmospheric waves at higher frequencies interact with the surface field and excite various MHD waves.

Other mode parameters also vary with activity. Both the amplitudes and the life times of the modes are seen to decrease with increasing activity (Salabert \& Jiménez-Reyes 2006; Komm et al. 2000; Chaplin et al. 2003), in agreement with observations that active regions suppress the convection which excites the oscillations. This mechanism is also supported by the observation that the energy supply rate to the modes does not change with increasing surface activity but the damping increases (Salabert et al. 2007). The spatial and temporal pattern of the mode parameter shifts can be projected back onto the solar surface (Komm et al. 2002; How et al. 2002) and is seen to be highly correlated with the activity bands.

The continuous coverage also allows us to study changes in the frequencies on short time scales. Tripathy et al. (2007) analyzed all of the GONG and SOHO-MDI data in nine-day segments, substantially shorter than the standard 108-day GONG or 72-day MDI analyses. They found that the nine-day frequencies are also highly correlated with activity indices, with the correlation at low and high frequencies decreasing more rapidly than in the longer analyses. The low-frequency decrease is likely due to increased noise and poorer frequency resolution in the short time series which degrades the precision of the measurements. The high-frequency decrease may arise from the short lifetimes of the modes, which do not survive long enough to interact with the surface activity. Tripathy et al. (2007) also found that the sensitivity of the short-time scale frequency shifts to the magnetic field index is significantly higher in the ascending phase of the cycle compared to the descending phase. This may be related to the hysteresis seen in other solar activity indices.

\subsection{3-D MHD global simulations of solar internal dynamics}

The Sun's internal dynamics is very complex, involving nonlinear interactions between convection, turbulence, instabilities, rotation, shear, and magnetism. Being able to understand the intricate interplay between all of these processes is crucial if one wants to progress in our knowledge of the Sun and the stars for which the Sun is our only spatially resolved example. Numerical simulations are thus becoming more and more useful for tackling this challenging problem. We briefly report here on the current status of 3-D global MHD simulation of solar internal magnetohydrodynamics obtained with the 3-D MHD ASH code (Clune et al. 1999; Miesch et al. 2000; Brun et al. 2004). In particular we wish to summarize the physical processes behind differential rotation, meridional circulation, and the solar magnetic activity and cycle.

The differential rotation of the Sun's convective envelope is peculiar since it is almost constant along a radial line at mid-latitude, a property which is counter intuitive, since it has been known for a long time that rotating fluids usually possess a cylindrical rotation profile (Taylor-Proudman theorem and columns, see Brun \& Toomre 2002). What can 

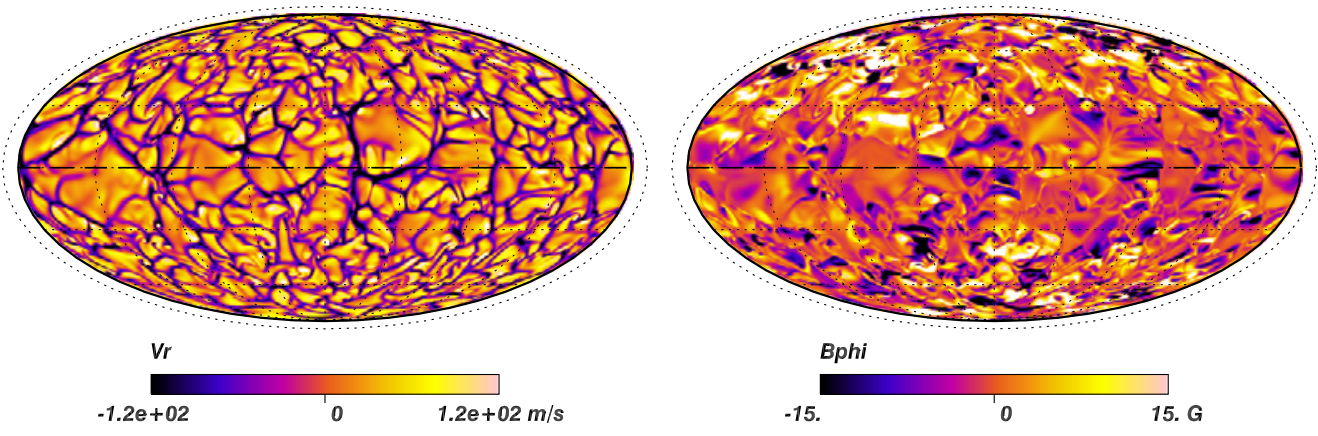

Figure 1. Snapshot using a Mollweide projection, of the radial velocity and toroidal field, near the top of the domain for the purely unstable magnetized convection model with a small magnetic Prandlt number $P m=0.8$. Typical field strengths are indicated, with dark tones corresponding to downward velocities and negative polarities. The dashed line indicates the equator.

3-D global models tell us about the processes leading to such a special rotation profile? A careful study of the redistribution of the angular momentum in a turbulent convection shell indicates that Reynolds stresses, i.e., non-linear correlations between the radial and latitudinal velocities with the azimuthal one) are the key players in transporting the angular momentum from the polar region toward the equator thus accelerating the equatorial region. The ASH simulations also show that a strong thermal wind is associated with this pronounced differential rotation, with associated temperature contrast of order few $\mathrm{K}$. The conical shape is the result of a subtle latitudinal heat and angular momentum transport that favor a fast equator and slow poles and a weak pole to equator temperature contrast. A small change of the amplitude of this temperature contrast is found to have a drastic effect on the resulting profile of angular velocity, at $3 \mathrm{~K}$ the profile is cylindrical, at $13 \mathrm{~K}$ it is almost horizontal, with a value of $10 \mathrm{~K}$ giving the best agreement with helioseismic inversion (Miesch et al. 2006). Such a very delicate nonlinear balance between heat and angular momentum transport, is likely to be at the origin of the current observed differential rotation. As a consequence, small change in the parameters of convection models lead to a large range of differential rotation profiles (Brun \& Toomre 2002). It thus remains to be seen, as the level of turbulence of the simulations are increased to become more realistic, how anisotropic nonlinear heat transport could finally setup a temperature contrast of $10 \mathrm{~K}$ found to reproduce reasonably well the observations.

The meridional circulation driven by the convection is found to be much weaker than the differential rotation, since it can be considered as a small departure from geostrophic balance. In the current 3-D global simulations, meridional flows are found to be multicellular both in latitude and radius, even in the case possessing a conical solar-like profile (Miesch et al. 2006). A recent study by Jouve \& Brun (2007) indicates that such multicellular meridional flows will modify the butterfly diagram and decrease the cycle period in 2-D mean field models. If such complex meridional flows were to persist over many years in the Sun, they will either lead to a modified butterfly diagram or push the solar dynamo community to reconsider standard Babcock-Leighton solar dynamo models (Dikpati et al. 2004), as in the mid-1980's the inversion of the angular velocity lead the community to modify the standard $\alpha-\omega$ model. Clearly, inverting the meridional flow down to the tachocline is rather urgent if one wants to progress in our understanding of the internal dynamics of the Sun.

Concerning 3-D modeling of solar magnetism, today the most accepted scenario to explain the Sun's large variety of dynamical phenomena rests on the operation of an 
internal dynamo in the highly-turbulent-convective solar envelope and at its base, in the tachocline (Parker 1993; Dikpati et al. 2004; Brun et al. 2004; Jouve \& Brun 2007). This dynamo is thus operating at different location and physical scales and organize the large-scale field such as to make it cyclic. High-resolution computations performed with the ASH code (Brun et al. 2004), confirmed that dynamo action in a rotating turbulent convection zone generates magnetic fields at all scales present in the velocity field and that the field filled up the sphere leading to the formation of an $\ell=1$ dipolar component. This dipolar field contains about $1 \%$ of the total magnetic energy generated in the convection zone which is mostly found in non axisymmetric field, and its amplitude is about $10 \mathrm{G}$. It is found to oscillate irregularly with a pseudo-period of about 400 days, almost a factor of ten faster than the real solar 11-yr cycle. Further we do not find in our purely unstable simulations strong toroidal mean magnetic field, as the cyclic emergence of sunspot on the solar surface suggest. A simple explanation is that the highly dynamical convection zone is not able to generate strong flux tube like structures locally. The fast, irregular reversal and the weak, toroidal mean field together point to the necessity or having a stable layer, where both the dynamical time scale is slower and the winding up of a poloidal field into strong toroidal structure is made easier. This has thus led to the incorporation of the role of the tachocline in a 3-D MHD simulation of the global solar magnetism (Browning et al. 2006). The results are encouraging since having a tachocline generates much stronger mean toroidal fields than previously found (by a factor found to be between 10 to 100), that possess the antisymmetry observed in sunspots between the two hemispheres (Hale's law). Also the presence of a strong field in the stable layer seems to have a stabilizing influence of the reversal of the weak poloidal field generated above in the turbulent convection zone, suggesting that the solar activity can be controlled by processes occurring rather deep in our star. A recent work by Brun \& Zahn (2006)as also demonstrated that if a deep, fossil magnetic field exists in the solar radiative interior, this field is most likely in a mixed poloidal/toroidal configuration rather than just in a simple dipolar shape. Indeed they confirmed the pioneering work of Tayler in the mid1970's that showed that a purely poloidal or toroidal magnetic field will become unstable respectively to high $m$ and $m=1$ instabilities. This complex topology and evolution of the inner field, and its coupling with the dynamo field of the tachocline can potentially lead to interesting modulation of the solar magnetic cycle. It is thus crucial to consider the Sun has a whole rather than to split it into its radiative, convective, and external parts. The time has come to develop a fully integrated model of the solar dynamics that coupled as much as possible all the fascinating dynamics occurring in its interior, transition layers and at its surface (Brun 2007).

\subsection{Solar interior dynamics and their temporal variation}

Turning to the internal solar dynamics, Howe et al. (2000) found a 1.3-yr oscillation in the rotation rate in the tachocline. While Basu \& Antia (2001) were unable to reproduce the result, it remains intriguing and further work by How et al. (2006b) have shown it may be intermittent, since it apparently ceased after solar cycle 23 maximum in 2002 . Continued observations will be needed to determine if it reappears in the ascending phase of Cycle 24, which is just now starting in August 2006.

Global helioseismology has also revealed that the so-called torsional oscillation seen on the surface for the last 30 years extends almost all of the way through the convection zone (How et al. 2005; Vorontsov et al. 2002). There is evidence that this zonal flow originates deep in the convection zone and then rises to the surface over the course of the cycle (How et al. 2005). The current temporal evolution of the flow can be described by the sum of an 11-yr and an 11/2-yr period sinusoid, and the near-surface helioseismic 
inferences of the flow are very highly correlated with the surface observations (How et al. 2006a). Finally, the helioseismic observations show the zonal flow associated with Cycle 24 began to migrate from the poles towards the equator in 2002, some four years before the first Cycle 24 active region was seen. This supports the idea of an extended solar cycle, as observed in coronal green-line observations (Altrock \& Howe 2004).

\subsection{A flux-transport dynamo model}

Recent work has aimed at producing a non-kinematic flux-transport dynamo model to address the non-linear saturation through Lorentz force feedback as well as cycle variations of differential rotation and meridional flow. To this end one combines the differential rotation and meridional flow model developed recently by Rempel (2005) with a fluxtransport dynamo similar to the models of Dikpati \& Charbonneau (1999) and Dikpati \& Gilman (2001).

The differential rotation model utilizes a meanfield Reynolds-stress approach that parametrizes the turbulent angular momentum transport $(\Lambda$-effect, Kitchatinov \& Rüdiger 1993) leading to the observed equatorial acceleration. The tachocline is forced through a uniform rotation at the lower boundary of the computational domain. A meridional circulation, as required for a flux-transport dynamo, follows self-consistently through the Coriolis force resulting from the differential rotation. The computed differential rotation and meridional flow are used to advance the magnetic field in the flux-transport dynamo model, while the magnetic field is allowed to feed back through the macroscopic Lorentz force $\langle\boldsymbol{J}\rangle \times\langle\boldsymbol{B}\rangle$ (the contribution of the fluctuating part $\left\langle\boldsymbol{J}^{\prime} \times \boldsymbol{B}^{\prime}\right\rangle$ is not well known and neglected here).

It is found that the dynamo saturates through a reduction of the mean differential rotation when the toroidal field strength at the base of the convection zone reaches values around 1 to $1.5 \mathrm{~T}$. The feedback on the meridional flow remains weak for these values, meaning that the equatorward transport of toroidal field at the base of the convection zone (crucial ingredient in a flux-transport dynamo) is only marginally affected.

The feedback of the macroscopic Lorentz force on differential rotation leads to a poleward propagating branch of torsional oscillations in agreement with observations in terms of amplitude and phase; however, the equatorward propagating low-latitude branch requires additional physics. Parameterizing the idea proposed by Spruit (2003) that the low-latitude torsional oscillation is a geostrophic flow caused by increased radiative loss in the active region belt (due to small scale magnetic flux) leads in a model to a surface oscillations pattern in good agreement with observations (see Fig. 2). As a side effect the cooling produces close to the surface an inflow into the active region belt of a few $\mathrm{m} \mathrm{s}^{-1}$ in amplitude, which has been observed through surface Doppler measurements by Komm at al. (1993); Komm (1994), and with local helioseimology by, e.g., Gizon (2004) and Zhao \& Kosovichev (2004). Around $50 \mathrm{Mm}$ depth we find an outflow with an amplitude reduced by one order of magnitude (due to the increase in density). Recently Gizon \& Rempel (2006) found observational support for such an outflow on a qualitative level.

Further details and properties of the solution of the coupled-differential-rotation dynamo model can be found in Rempel (2005) and Rempel (2006).

\subsection{High-degree mode parameter determination}

An aspect of helioseismic data analysis that still requires improvement is the accurate determination of the mode parameters used in helioseismology, particularly at high degree. The inclusion of high-degree modes (i.e., $\ell$ up to 1000) has the potential to improve dramatically the inference of the sound speed and the adiabatic exponent $\left(\Gamma_{1}\right)$ in the outermost 2 to $3 \%$ of the solar radius (Rabello-Soares et al. 2007), a region of great 


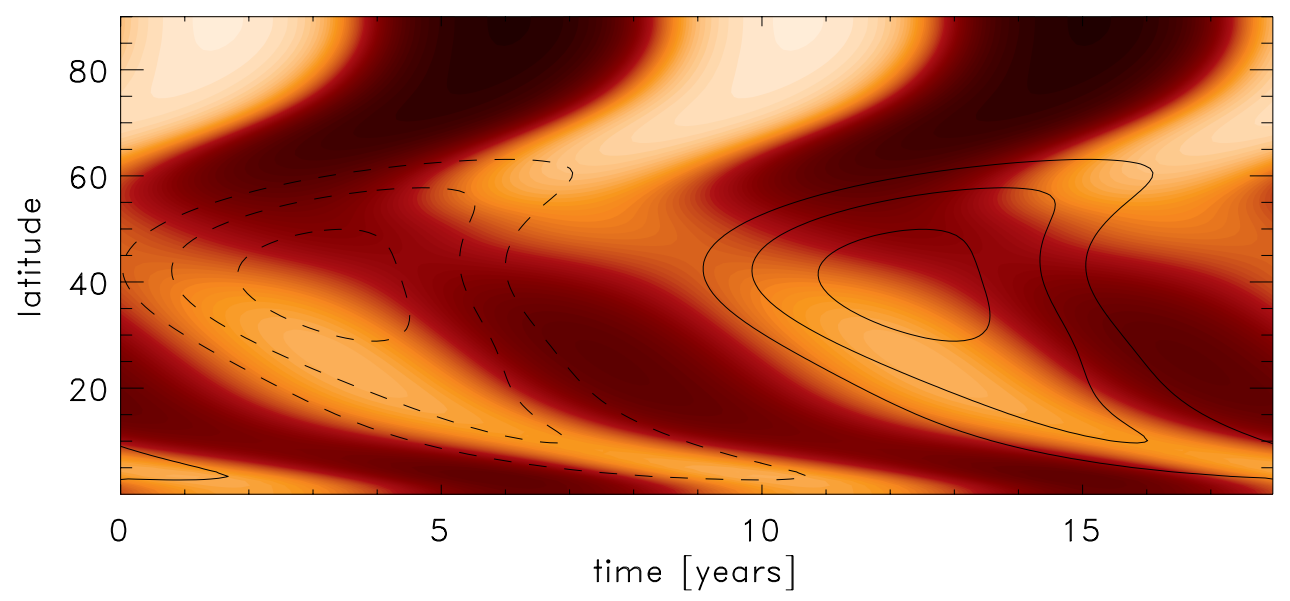

Figure 2. Surface pattern of torsional oscillations in a model considering macroscopic Lorentz force feedback and cooling of the active region belt. The contour lines indicate the toroidal field at the base of the convection zone. Bright colors refer to faster rotation, dark colors to slower rotation.

interest. However, the spherical harmonic spatial filtering is not orthonormal over less than the full Sun and the resulting spatial leaks get closer in frequency as the degree of a mode increases. As a result, the high-degree individual modes blend into ridges which will mask the true underlying mode parameters. This has so far prevented the estimation of unbiased mode parameters at high degrees using global helioseismology. As discussed by M.C. Rabello-Soares, in order to recover the mode characteristics, a very good model of the relative amplitude of all the modes that contribute to the ridge is needed, which requires a very good knowledge of the instrumental properties. Such a model of the relative amplitudes is called a 'leakage matrix'.

Rabello-Soares and collaborators have incorporated most of the observational and instrumental effects of the $\mathrm{SOHO}$-MDI instrument important to high-degree analysis into a new analysis. Compared to their previous work (Korzennik et al. 2004), they include the following effects: correct instantaneous plate scale, radial image distortion, CCD tilt $\left(\sim 2^{\circ}\right)$ with respect to the optical axis, effective $P$ angle $\left(0.2^{\circ}\right)$ and Carrington elements correction of $0.1^{\circ}$ (Giles 1999). They have analysed variations of the central frequencies for $100 \leqslant \ell \leqslant 1000$ caused by the solar activity cycle. They showed that the high-degree changes during solar cycle 23 are in good agreement with the medium-degree results, except for years when the instrument was highly defocused (1996 to 1998) which were excluded from the analysis.

Although the correlation of the frequency changes with the solar cycle has been known for some time, its physical origin has been a matter of debate. The analysis of its behaviour will hopefully help in its understanding. Rabello-Soares et al. observed that the $p$ modes and $f$ (fundamental) modes behave differently, which suggests different physical effects. Their results confirm that the $p$-mode frequency shift scaled by the relative mode inertia is a function of frequency alone (see Rabello-Soares et al. 2007). The scaled frequency shift increases until $4.8 \mathrm{mHz}$. At higher frequencies, the frequency variation does not appear correlated with solar activity. 


\subsection{The search for solar $g$-modes}

A detailed knowledge of the interior of the Sun has been obtained by means of precise measurements of its acoustic eigenmodes and the subsequent structural inversions, providing the stratification of crucial variables like the sound speed down to $0.05 \mathrm{R}_{\odot}$ (Turck-Chièze et al. 2001). The radial profile difference of the observed sound speed with the one extracted from the solar evolution model depends strongly on the physics used. The seismic model which has been built to reduce this difference to practically zero in the radiative zone enables to predict neutrino fluxes in excellent agreement with their detection (Couvidat et al. 2003a). However, the acoustic modes are less sensitive to other structural variables such as the density. In this case, the agreement with the models is still poor in the radiative zone. Moreover, the dynamical properties of the nuclear core are practically unknown. Effectively, due to the limitation in the number of acoustic modes penetrating in this region, the solar rotation profile is very uncertain below $0.2 \mathrm{R}_{\odot}$ (Chaplin et al. 1998; Couvidat et al. 2003a; García et al. 2004).

To improve our knowledge of the deepest layers of the Sun, the detection of gravity modes is required. These modes propagate in the radiative zone but are evanescent in the convective region and their expected surface amplitudes could be very small (Andersen 1996; Kumar et al. 1996). These modes have been intensively looked for since the 1980s. In the last ten years, two complementary approaches have been developed: to look for individual modes (relevant spikes in the Fourier spectrum of the observed signal) above a given statistical threshold (typically $90 \%$ confidence level) at frequencies above $15 \mu \mathrm{Hz}$; and to look for their predicted asymptotical properties at lower frequencies. The individual mode search has provided several $g$-mode candidates in SOHO-GOLF observations with more than $90 \%$ confidence level. In this study, multiplets instead of single peaks have been looked for (Turck-Chièze et al. 2004). The frequencies of such modes are extremely sensitive to the structural properties of the radiative zone (Mathur 2006) and their splittings provide information on the solar core rotation. But, today, there remains some ambiguity on the proper identification of the detected components in terms of their $\ell, m, n$ properties. Some scenarios have been proposed with consequences on the core dynamics (Turck-Chièze 2006a). The search for the asymptotic $g$-mode properties has been with us from the earliest days of helioseismology (summarized in Pallé 1991 and Hill et al. 1991) but using low-quality observational data sets (pre-space and prenetworks era). The asymptotic approach (where $n \gg \ell$ ) predicts a constant separation between the periods of adjacent $(n, n+1)$ gravity modes. Consequently, periodicities in the form of broad peaks are expected in the power spectrum (PS) of the power spectral density (PSD) expressed in period corresponding to the observed velocity time series. This year, a peak structure has been detected (see Fig. 3), with more than $99.86 \%$ confidence level (García et al. 2006), thanks to the high quality datasets available from SOHO-GOLF (García et al. 2005). The detailed analysis of such structure provides access to the dynamics of the inner core, in particular its rotation rate. At the present stage of this investigation, it would favour a core rotating faster than the rest of the radiative zone. If the detection of these $g$-mode signatures is confirmed, a very exciting future will be ahead of us to improve our knowledge of the dynamics of the deepest layers inside the Sun. Both analyses are fully complementary and we are encouraged to apply them to other instruments aboard $\mathrm{SOHO}$ and to improve once more our capability of detection. A new generation of instruments is necessary to reveal the complete dynamics of the core including its magnetic field. We are hoping to be able to improve the capability of intensity measurement at the limb (SODISM-PICARD) and to optimize the signal-tonoise ratio at these very low frequencies by reducing the solar noise (GOLFNG). This 


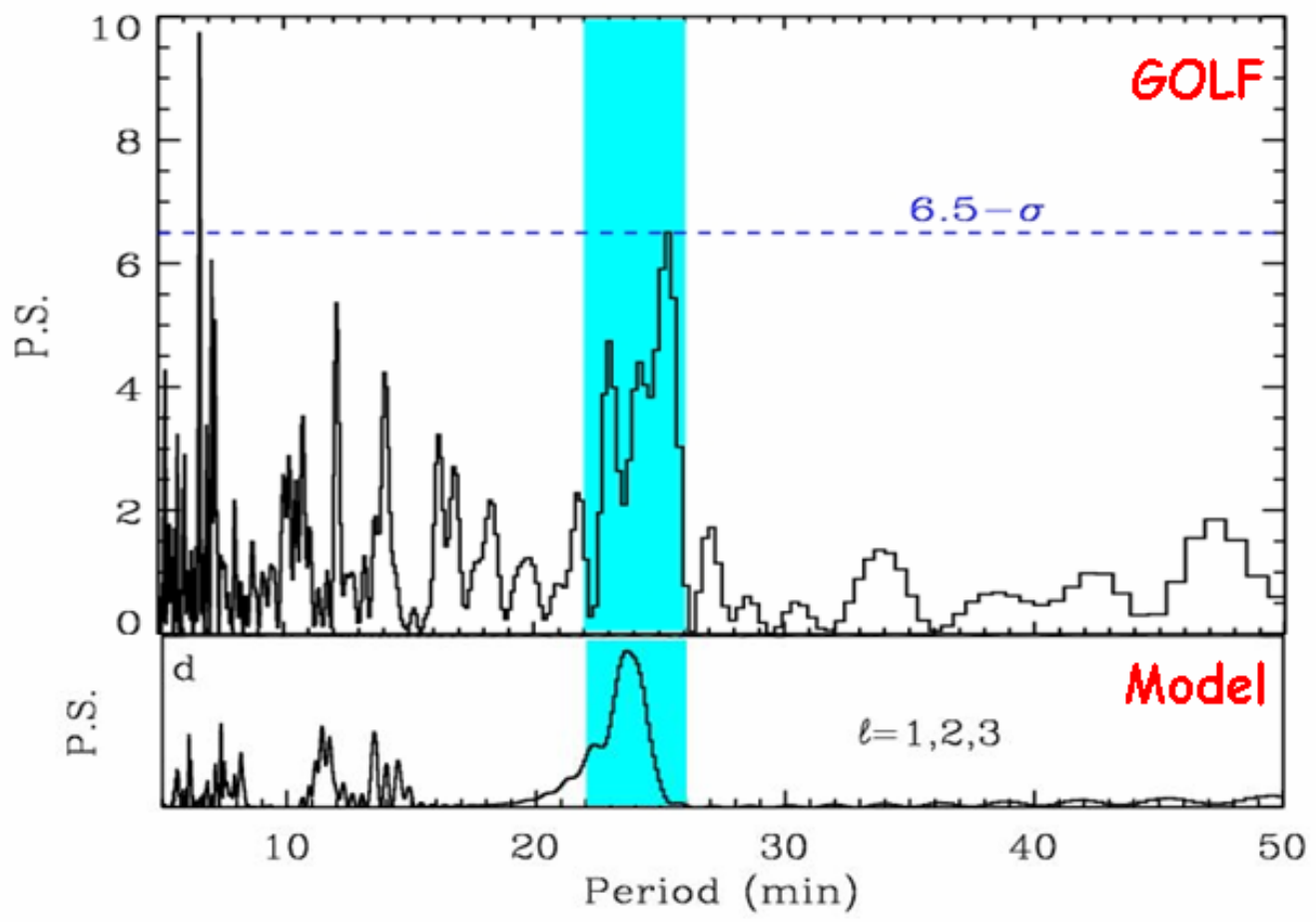

Figure 3. Top: Power spectrum of the power spectral density expressed in period computed from ten years of SOHO-GOLF velocity time series. Bottom: PS of the PSD from a model containing theoretical $g$-modes computed from the seismic model and using a rigid core rotation.

is the motivation of the DynaMICS (Dynamics and Magnetism from the Inner Core to the Chromosphere (ES Corona) of the Sun) mission (Turck-Chièze et al. 2006c), which aims to make use of a small satellite with the GOLFNG instrument (Turck-Chièze et al. 2006b ) while Solar Dynamics Observatory (SDO) and SODISM-PICARD are operating, as well as possibly as part of a bigger platform within the ESA Cosmic Vision 2015-2025 programme (Turck-Chièze et al. 2005).

\subsection{Solar interior dynamics: angular momentum transport by gravity waves}

It is now well established that the interior of the Sun is rotating more or less at the same rate as the average rotation rate of the surface convection zone. We also know that, in the past, the Sun was rotating much faster; the interaction of the Sun's lost mass with the outer magnetic fields explains the spin-down of the convection zone, but there remains the need for a very efficient mechanism to carry angular momentum from the radiative core to the surface convection zone.

Internal gravity waves excited at the base of the convection zone may be an important source of angular momentum redistribution, since they take momentum from the region where they are excited and deposit it where they are damped. These waves have low frequencies (around $1 \mu \mathrm{Hz}$ ) and are the propagating counterparts of the higher-frequency $g$-modes that form standing waves. Talon et al. (2002) showed how differential filtering exerted by an asymmetrical-shear-layer oscillation (itself related to the damping of high-degree internal gravity waves) favors the penetration of low-degree, low-frequency retrograde waves that may deposit their negative angular momentum in the deep interior, causing the spin-down of the core. 

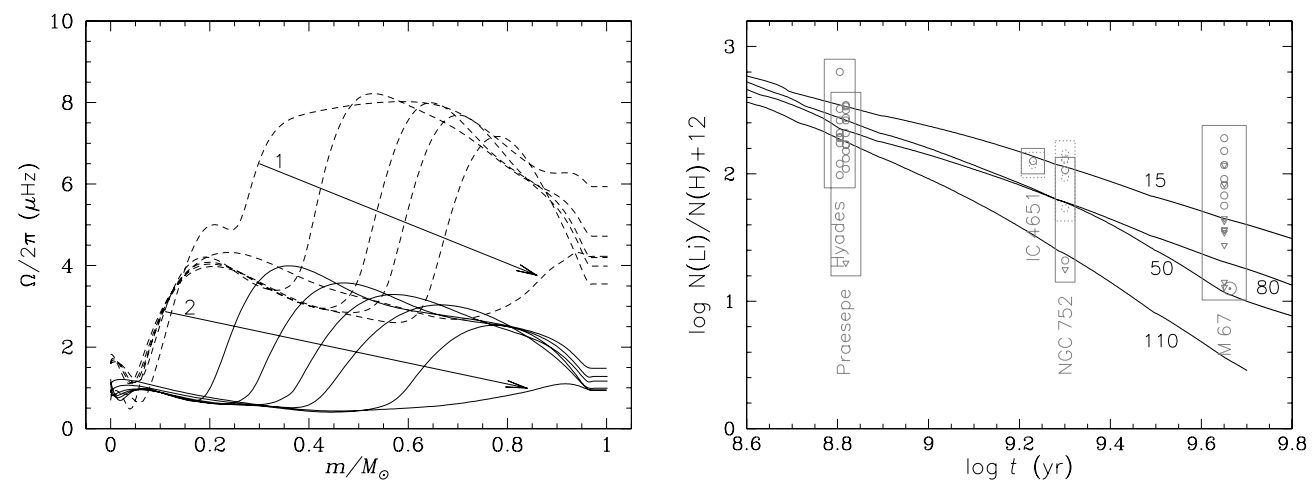

Figure 4. Left: Evolution of the solar rotation profile under the effect of surface braking, meridional circualtion, shear turbulence, and internal gravity waves (ages: 0.2, 0.21, 0.22, 0.23, $0.25,0.27,0.5,0.7,1,1.5,3,4.6 \mathrm{~Gy})$. Right: Evolution of the surface lithium abundance for various initial rotation rates (black lines) compared to measurements of $\mathrm{Li}$ in solar-mass stars in clusters of various ages (grey points and boxes). Adapted from Charbonnel \& Talon (2005).

Charbonnel \& Talon (2005) applied that model to an evolving solar-mass model in which the surface convection zone is slowly spun-down with time. Internal gravity waves then produce a front of 'slowness', that propagates from the core to the surface, resulting in a much reduced differential rotation at the solar age when compared with the one obtained using 'classical' rotational-mixing models (Fig. 4). The time-evolution of lithium destruction obtained in those models is consistent with Li measurements in solar-mass stars of various ages and in the Sun, using free parameters that are calibrated on massive stars.

\subsection{Solar asphericities}

As a different tool for analysis, solar-shape coefficients $\left(c_{n}\right)$, also referred to as asphericities, reflect the internal non-homogeneous mass distribution and non-uniform angular velocity (Fig. 5, left). A careful inspection of the curve $c_{n}(r)$ reveals two breaks, of which one is the signature of the tachocline. The second, located around $0.995 \mathrm{R}_{\odot}$ was dynamically studied through the $f$ modes, which probe the physical changes just below the photosphere (Lefebvre \& Kosovichev 2005).

The study of asphericities, directly linked with solar gravitational moments is not only crucial for solar physics, but also for astrometry (when computing light deflection in the vicinity of the Sun), celestial mechanics (relativistic precession of planets, planetary orbit inclination and spin-orbit couplings) and for future tests of alternative theories of gravitation (correlation of $J_{2}$ with Post-Newtonian parameters).

According to the temporal variation of the $f$-mode frequencies, it is found that the very near solar surface is stratified in a thin double layer, interfacing the convective zone and the surface. This 'leptocline' is the seat of many phenomena: an oscillation phase of the seismic radius, together with a non-monotonic expansion of this radius with depth (Fig. 5, right), a change in the turbulent pressure (Fig. 6), likely an inversion in the radial gradient of the rotation velocity rate at about $50^{\circ}$ in latitude, and the cradle of hydrogen and helium ionisation processes (Lefebvre et al. 2006).

\section{Local helioseismology, magnetic activity and mode physics}

Local helioseismology has developed rapidly in the last few years, offering a threedimensional view of the Sun. Thanks to high-resolution, uninterrupted observations par- 

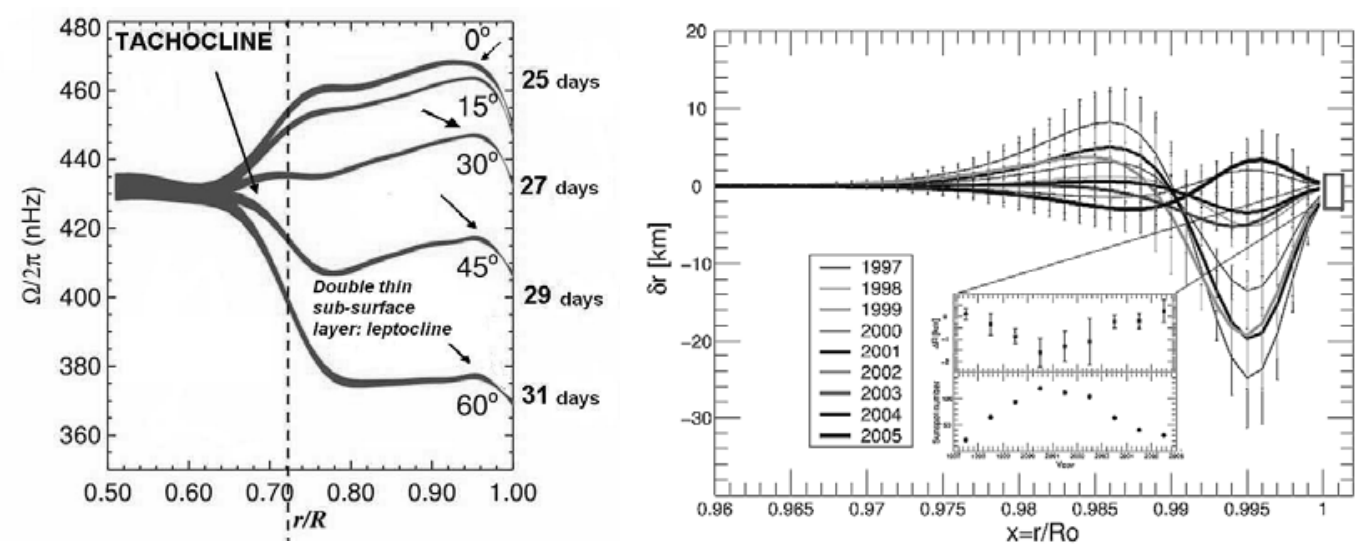

Figure 5. Left: The velocity rotation rate indicates a break near the surface, from Howe (2003). Right: $f$-mode analysis show a non-monotonic expansion of the solar radius with depth and a phase change with activity. From Lefebvre \& Kosovichev (2005).

ticularly from MDI onboard $\mathrm{SOHO}$ and $\mathrm{GONG}++$, it has become possible to image convective structures, sunspots, and active regions below the solar surface. Progress was reviewed by L. Gizon and A.G. Kosovichev. A particular challenge for the correct interpretation of local-helioseismic data in active regions, for example, is the theoretical modelling of the interaction of waves with the magnetic field (see below). Another important area of advance is in numerical simulations pertinent to understanding the flows of the upper convection zone and the interaction of acoustic waves with these flows. R. Stein reviewed work on a realistic solar surface convection simulation on supergranulation scales (48 Mm wide by $20 \mathrm{Mm}$ deep), whose duration is currently $48 \mathrm{hr}$. There is a rich spectrum of $p$-modes excited in the simulation, and the data set is available for studying solar oscillations and local helioseismic inversion techniques.

\subsection{Time-varying component of the meridional circulation}

At the Sun's surface, the average motion in meridional planes is from the equator to the poles with a maximum amplitude of $10-20 \mathrm{~m} \mathrm{~s}^{-1}$ near $25^{\circ}$ latitude. Temporal variations in the longitudinal average of the meridional flow have been reported by several authors using direct Doppler measurements or correlation tracking of small photospheric magnetic features. Only local helioseismology can provide measurements of the subsurface meridional circulation (Giles et al. 1997). It is known that the time-varying component of the meridional flow with respect to a long-term average does not exceed $\pm 5 \mathrm{~m} \mathrm{~s}^{-1}$ and is consistent with a small near-surface inflow toward active latitudes (Basu \& Antia 2003; Gizon 2004; Zhao \& Kosovichev 2004; González Hernández et al. (2006); Komm et al. 2006) and an outflow from active latitudes at depths greater than $20 \mathrm{Mm}$ (Chou \& Dai 2001; Beck et al. 2002; Chou \& Ladenkov 2005).

Recently, Gizon \& Rempel (2006) obtained independent measurements of the temporal variations of the meridional circulation near the solar surface and at a depth of about $60 \mathrm{Mm}$. These measurements confirm the previous observations listed above. In addition, the observations were compared with Rempel's theoretical model (Section 1.4) based on a flux-transport dynamo combined with a geostrophic flow caused by increased radiative loss in the active region belt, according to Spruit's (2003) original idea. The model is qualitatively consistent with the observations, in particular concerning the phase of the solar-cycle variations of the flows near the surface and $60 \mathrm{Mm}$ below. Near the surface, the model is agreement with the data: the relative amplitudes of torsional oscillation 


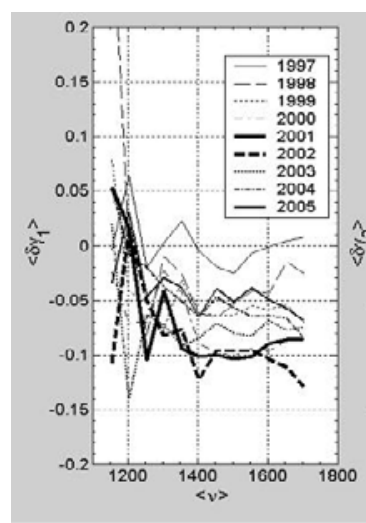

$\gamma_{1}<0$ antiphase

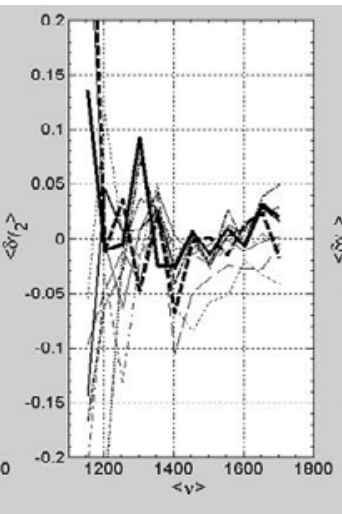

$\gamma_{2}$ positive trend

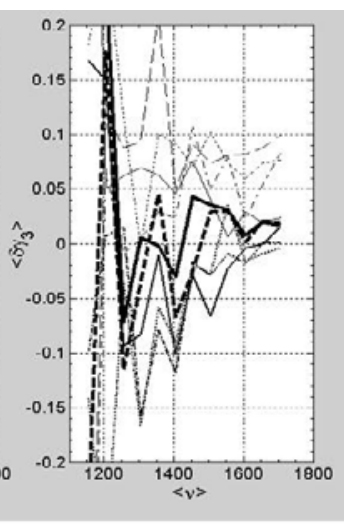

$\gamma_{3}$

Figure 6. Three first asphericities parameters $\gamma$, i.e., even- $a$ coefficients of $f$-modes. The layer around $0.995 \mathrm{R}_{\odot}$ is the seat of numerous physical changes. From Lefebvre et al. (2006).

and the time-varying component of the meridional flow and their relative phase are well reproduced. Deeper in the interior, it appears that the model underestimates the amplitude of the time variations of the meridional flow by nearly an order of magnitude; however, the flow variation is in antiphase to the surface flow as seen in the data. Overall, it is fair to say that the model is encouraging. We note that a local treatment of the regions of strong magnetic-field concentrations (sunspots and active regions) might be necessary to obtain a better match between the model and the data (see Gizon 2004).

\subsection{Magnetohelioseismology}

It is well known that sunspots absorb energy from and increase the phase speed of $f$ - and $p$-modes incident upon them (Braun 1995). When this was first observed it was hoped that this information could be used to probe the subsurface and internal structure of sunspots. However, interpretation of the observations was difficult. It was only recently that the likely mechanism causing each of these effects was identified (although the idea was originally put forward some time ago by Spruit 1991). Models based on the conversion of acoustic oscillations to magnetohydrodynamic waves within sunspots, although simplistic in several respects, can now explain the absorption and phase speed changes simultaneously (Cally et al. 2003; Crouch et al. 2005). This confirms that mode conversion is the most promising absorption mechanism with wave energy channeled both up and down the magnetic field inside sunspots. It was also discovered that field inclination is a vital ingredient for mode conversion to work efficiently (Crouch \& Cally 2003, 2005).

Ray conversion theory, as developed by Cally (2006) and Schunker \& Cally (2006), has provided several new insights into the problem of how solar oscillations interact with magnetic field. Under that formalism, mode conversion is evident as ray splitting. Ray conversion theory has shown that fast ray travel times can be reduced by several minutes in comparison to the nonmagnetic acoustic ray in the same solar model, with obvious helioseismic implications. In addition, it has also shown that fast to slow mode conversion is very sensitive to the angle of attack that the wavevector makes with the magnetic field at the equipartition layer, where the sound speed and Alfvén speed coincide. A major consequence of this insight is that the magnetic field acts as a filter, preferentially allowing through acoustic signal from a narrow range of incident directions. This directional filtering effect has been confirmed by three dimensional wave mechanical calculations, 
which show that the upward flux of acoustic energy is indeed greatest when the propagation direction is aligned with the magnetic field (which is analogous to a fine attack angle).

With the most recent wave mechanical models now accounting for wave propagation in both the solar interior and atmosphere, we can predict how strong magnetic fields influence the polarisation of the velocity signal at heights where it is typically observed (such as at the formation height of the $676.8 \mathrm{~nm}$ Ni I spectral line used by $S O H O$-MDI and GONG). It turns out that the eccentricity and inclination of the principal axis of the velocity ellipse are very sensitive to the ratio of sound and Alfvén speeds at a given height. At great height in the atmosphere, where the Alfvén speed greatly exceeds the sound speed, the fast and Alfvén waves are evanescent; whereas the slow mode (which dominates the velocity signal at these levels) is a field guided travelling acoustic wave and causes the velocity ellipse to be highly eccentric and field aligned. At lower levels, where observations are typically made (and the sound and Alfvén speeds are comparable), the fast and Alfvén waves also contribute to the velocity signal and the slow mode is less dominant, resulting in a more complex pattern. In the near future we aim to compare these model predictions with observations of the surface velocity (e.g., Schunker et al. 2005).

\subsection{Seismic diagnostics inferred from amplitudes of stochastically-excited modes}

Solar-like oscillations in main-sequence stars, as well as in some red giant stars, are excited by turbulent convection in the strongly superadiabatic outermost convective-zone boundary layer. From the measurement of the amplitude and line-width of resonant modes, it is possible to infer the power supplied to the mode by turbulent convection, which in turn provides a unique probe of turbulent convection in stars. Such measurements have been used in helioseismology for some time now (e.g., Goldreich \& Keeley 1977; Balmforth 1992; Samadi \& Goupil 2001; Chaplin et al. 2005), and recently stellar measurements have become available from $\alpha$ Cen A from the ground (Bedding et al. 2004; Kjeldsen et al. 2005) and from space (WIRE) (Schou \& Buzasi 2001; Fletcher et al. 2006). Recent developments have been reviewed by Houdek (2006) and Samadi et al. (2007).)

The model of Samadi \& Goupil (2001), with the improvements proposed by Belkacem et al. (2006b), was presented in depth. Such a model requires a prescription of the temporal-correlation between the turbulent elements, which is obtained from a 3D simulation of the upper part of the solar convective zone, e.g., Samadi et al. (2003a). At large scales, this seems to be better fitted by a lorentzian than a gaussian, and the lorentzian fit appears to also agree better with the excitation rates inferred from helioseismic observations by Chaplin et al. (1998). The question remains as to whether this result remains valid for other stars. The model also requires a prescription for the fourth-order moments involving the entropy fluctuations and the turbulent velocity. The quasi-normal approximation, which consists in splitting the fourth-order moments into the product of two second-order moments, provides a convenient starting point, but it is significantly biased in the solar convective zone (Belkacem et al. 2006a) leading to an under-estimate of the $p$-mode excitation rate. More sophisticated closure models can be used, for example the two-scale mass-flux model (Gryanik \& Hartmann 2002) which takes the asymmetries in the medium into account, but it is only applicable for quasi-laminar flows. Belkacem et al. (2006a) have generalized the approach by taking the turbulent properties of the medium into account, introducing plumes dynamics following Rieutord \& Zahn (1995). As shown by Belkacem et al. (2006b), calculations based on this new closure model increase the contribution of the Reynolds stress to the excitation rates of the solar modes, and when the additional contribution due to entropy fluctuations is included, the new theoretical 
calculations fit rather well the maximum in the solar mode excitation rates derived by Baudin et al. (2005). The mode amplitudes and lifetimes from a significant sampling of stars that should be forthcoming from CoRoT will enormously improve the observational contraints on these models, and help us better understand the exitation of solar modes as well.

\section{A new era of asteroseismology}

Asteroseismology is at a particular exciting juncture with excellent new ground-based observations and with the recent launch of the satellite mission CoRoT. One mission already successfully launched and producing exciting results is MOST, which was reviewed by the mission's PI, J. Matthews. Much of what was presented at the meeting is summarized below. Solar-like oscillations have now been detected in quite a few main-sequence stars and sub-giants, as well as in red giants. One goal of asteroseismology is to study the internal rotation of other stars, as has been achieved for the Sun by helioseismology. M.-J. Goupil reviewed the challenges of seismic detection of rotation in other stars, and illustrated these with the results from a Hare-and-Hounds excercise carried out by the CoRoT team.

\subsection{An introduction to asteroseismology}

Helioseismology has provided very detailed and precise information about one specific star, the Sun. This has served as a crucial test of the theory of stellar internal structure and dynamics and has raised new challenging questions, such as the evolution of the rotation rate in the solar radiative interior, the origin of the differential rotation in the solar convection zone and, recently, the consequences of the new determinations of the solar surface abundances. However, studies of just one star, regardless of their quality, are evidently insufficient as a basis for understanding the broad range of phenomena occurring in stars of all masses over their eventful lives. Fortunately, stars throughout the HR diagram, from red supergiants to white dwarfs, show pulsations and hence offer at least some possibilities of asteroseismic studies.

In many cases, particularly near and below the main sequence, a sufficient number of modes are observed to allow, at least in principle, detailed constraints on stellar properties. Accurate determinations of stellar masses, radii and evolutionary states are of obvious interest; however, a more fundamental goal is to obtain data of sufficient quality to allow tests of the physical properties of stellar interiors and indications of the required improvements to our understanding of these properties.

Two general classes of modes are observed in stars. In $p$-modes, the dominant restoring force is pressure and the modes have the nature of standing acoustic waves. Such modes dominate the observed spectrum of solar oscillations. In $g$-modes, which are essentially standing internal gravity waves, buoyancy dominates the restoring force; this obviously requires departures from spherical symmetry and hence $g$-modes are only found with non-zero spherical harmonic degree $(\ell)$. In unevolved stars there is a clear separation between these two classes. However, in evolved stars with strong internal gradients in the chemical composition or large gravitational acceleration in a compact core, the characteristic buoyancy frequency of gravity waves becomes so high that the modes may have a mixed nature, behaving like a $g$-mode in the deep interior of the star and a $p$-mode in the external layers. Such mixed modes have very considerable diagnostic value.

For the foreseeable future almost all observations of stellar oscillations will take place in light integrated over the stellar disk. This strongly suppresses modes of degree $\ell$ exceeding three and hence the data are dominated by low-degree modes; this is particularly 


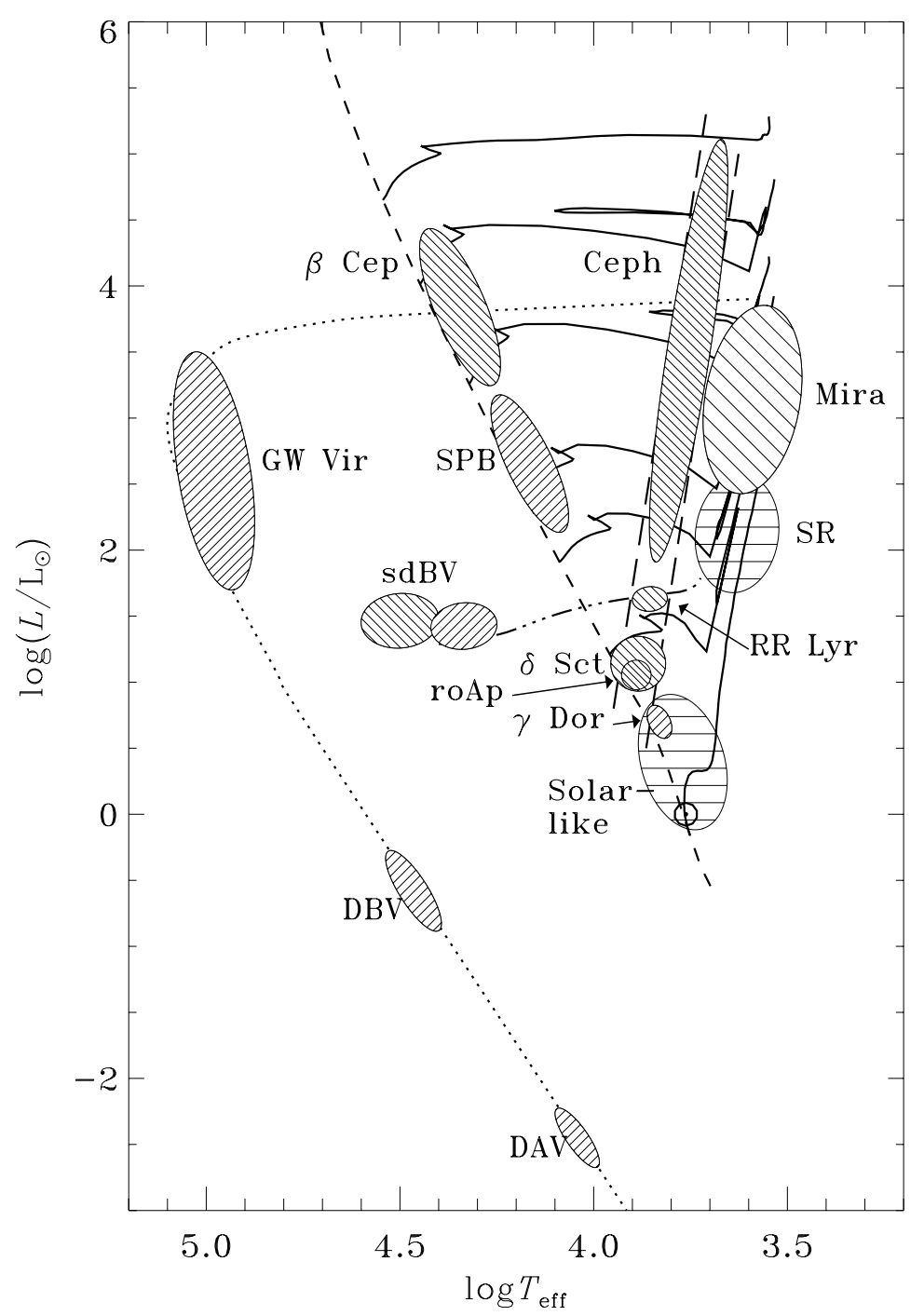

Figure 7. Hertsprung-Russell diagram showing the location of the principal types of asteroseismic variables stars discussed below.

true for solar-like oscillations where the stochastic excitation leads to true amplitudes that on average are largely independent of $\ell$. Compared with the extensive solar data this is obviously a strong restriction; however, fortunately, amongst the $p$-modes it is precisely the low-degree modes which penetrate most deeply into the star and hence provide information about its core properties.

Which modes are actually excited to observable amplitudes in a given star obviously depends on the relevant excitation mechanisms. A mode can either be self-excited or intrinsically damped but excited by forcing external to the mode. In the former case a critical region of the star operates as a heat engine, being heated in the compressional phase of the oscillation and cooled in the expansion phase. Such a region is typically associated with specific features in the opacity $(\kappa)$. To cause overall excitation the region 
has to be placed at the appropriate depth within the star. This constraint leads to relatively well-defined instability areas in the HR diagram. In the Cepheid instability belt, which extends to the $\delta$ Scuti stars near the main sequence, the opacity feature is associated with the second ionization of helium. An opacity feature at higher temperature, related to iron-group elements, causes instability in B-type stars near the main sequence and in subdwarf B stars on the extreme blue end of the horizontal branch. Convection may also cause heat-engine instability, typically at long periods; this is the case in the $\gamma$ Doradus stars and some of the pulsating white dwarfs.

An unstable mode grows exponentially with time, according to linear theory. The final amplitude is determined by nonlinear effects. For large-amplitude pulsators such as Cepheids, which typically show a single mode, amplitude limitation probably occurs through a saturation of the driving mechanism when the amplitude becomes sufficiently high. In stars near the main sequence, where many modes are typically excited, the distribution of observed amplitudes amongst the unstable modes is generally highly irregular, and no definite mechanism determining this distribution has so far been identified. Also, the irregular selection of observable modes often greatly complicates the identification of the observed modes with modes of stellar models.

In most stars on the cool side of the Cepheid instability strip, including the Sun, the modes appear to be linearly stable, and the observed oscillations are caused by stochastic excitation, driven by near-surface convection of such stable modes. In this case the mode amplitudes are determined on average by the balance between the energy input from convection and the damping rate. The result is a typical distribution of amplitude, such as observed in the Sun where the largest amplitudes are found near periods of five minutes. Within this overall amplitude envelope most modes are excited, although with varying amplitudes due to the stochastic nature of the excitation. As a result it is generally possible to identify the observed modes, using also the typically regular structure of the frequency spectrum. Together with the generally substantial number of observed modes this makes solar-like oscillations of great interest to asteroseismology.

\subsection{Observational results for solar-type stars}

Observations of solar-like oscillations are accumulating rapidly, and measurements have now been reported for several main-sequence, subgiant, and giant stars. The following list includes the most recent observations and is ordered according to decreasing stellar density (which also corresponds to a decreasing large frequency separation (between modes of different radial order $n)$ :

- $\tau$ Cet (G8 V): T.C. Teixeira et al. (in prep.)

- 70 Oph, a visual binary: Carrier \& Eggenberger (2006)

- $\alpha$ Cen A and B (see below)

- $\mu$ Ara, a planet-hosting star: Bouchy et al. (2005)

- HD 49933, a potential CoRoT target: Mosser et al. (2005)

- $\beta$ Vir (F9): Martić et al. (2004b); Carrier et al. (2005b)

- Procyon A (see below)

- $\beta$ Hyi, a G2 subgiant: Bedding et al. (2007)

- $\delta$ Eri, a K0 subgiant: Carrier et al. (2003)

- $\eta$ Boo (see below)

- $\nu$ Ind, a metal-poor subgiant: Bedding et al. (2006); Carrier et al. (2007)

- $\eta$ Ser (K0 III): Barban et al. (2004)

- $\xi$ Hya (G7 III): Frandsen et al. (2002); Stello et al. (2006)

- $\epsilon$ Oph (G9 III): De Ridder et al. (2006) 
On the main sequence, the most spectacular results have been obtained for the $\alpha$ Cen system. In the A component, more than 40 modes have been measured, with angular degrees of $\ell=0$ to 3 - see Bouchy \& Carrier (2002) and Bedding et al. (2004). The mode lifetime is about 2-4 days and there is now evidence of rotational splitting from photometry with the WIRE satellite analysed by Fletcher et al. (2006) and also from ground-based spectroscopy (M. Bazot et al., in prep.). Meanwhile, nearly 40 modes have been measured in - see Carrier \& Bourban (2003) and Kjeldsen et al. (2005).

Oscillations in the bright G0 subgiant $\eta$ Boo were measured by two independent groups using ground-based spectroscopy: Kjeldsen et al. (2003) and Carrier et al. (2005a). Some of those frequencies were seen in spaced-based photometry by the MOST satellite, as reported by Guenther et al. (2005), but those authors also claimed to detect oscillations at very low frequencies, the reality of which remains controversial.

The star Procyon A also generated controversy when MOST data reported by Matthews et al. (2004) failed to reveal oscillations that were claimed from ground-based data. For the latter, see Martić et al. (2004a), Bouchy et al. (2004), and Claudi et al. (2005) for the most recent examples. However, Bedding et al. (2005) argued that the MOST non-detection was consistent with the ground-based data.

In the future, we expect further ground-based observations using Doppler techniques (for example, a multi-site campaign on Procyon has been organized for January 2007). The new spectrograph SOPHIE at l'Observatoire de Haute-Provence in France should be operating very soon (<http://www.obs-hp.fr/>). From space, the WIRE and MOST satellites continue to return data and we look forward with excitement to the results from CoRoT (launched December 2006) and Kepler (to be launched in 2008).

Although remarkable, the results already obtained on solar-like oscillations are not sufficient to allow the desired detailed study of the internal properties of the stars. Simulations have shown that a frequency precision of the order of $0.1 \mu \mathrm{Hz}$ is required for detailed investigations of stellar cores, e.g., through inversion. A similar precision is required to extract the signatures of the base of the convective envelope and hence determine its depth, as well as to infer the envelope helium abundance from the effect of helium ionization on the sound speed. Also, such a frequency resolution is required to reliably measure the rotational splitting caused by slow rotation as in the Sun. To achieve this precision and resolution observations extending over at least several months are required. Mode identification itself remains a significant challenge as well. Furthermore, it is of great interest to extend the observations towards lower frequencies; here solar-like oscillations have long lifetimes, allowing high precision in the frequency determination, and are more sensitive to features such as the helium ionization. On the other hand, the amplitudes of such modes tend to be low, placing stringent constraints on the allowable noise level, both observational and stellar.

\subsection{Theory developments: dynamics of stellar radiative zones}

With the dramatic advances of helioseismology and of asteroseismology, a coherent picture of the evolution of rotating stars and of their internal dynamical processes is needed. In fact, rotation, and more precisely differential rotation, has a major impact on the internal dynamics of stars. First, as is known from the theory of rotating stars, rotation induces some-large scale circulations, both in radiation and convection zones, which act to transport simultaneously the angular momentum, the chemicals but also the magnetic field by advection. In radiation zones, the large-scale circulation, which is called the meridional circulation, is due to the differential rotation, to the transport of angular momentum and to the action of the perturbing forces, namely the centrifugal force and the Lorentz force (cf., Busse 1982; Zahn 1992; Maeder \& Zahn 1998; Garaud 2002a; Rieutord 2006). Next, 
the differential rotation induces hydrodynamical turbulence in radiative regions through various instabilities: the secular and the dynamical shear instabilities, the baroclinic and the multidiffusive instabilities. In the same way that the atmospheric turbulence in the terrestrial atmosphere, it acts to reduce the gradients of angular velocity and of chemical composition and thus, it is modelled as a diffusive process (cf., Talon \& Zahn 1997; Garaud 2001; Maeder 2003). On the other hand, rotation has a strong impact on the stellar magnetism. For example, it interacts with turbulent convection in convective envelopes of solar-type stars (cf., Brun et al. 2004) to lead to a dynamo mechanism and, as it is expected from observations, to a cyclic magnetism. In radiation regions, it interacts with fossil magnetic fields where the secular torque of the Lorentz force and the magnetohydrodynamical instabilities such as the Tayler-Spruit instability and the multidiffusive magnetic instabilities have a strong impact on the transport of angular momentum and of chemicals (cf., Charbonneau \& MacGregor 1993; Garaud 2002a; Spruit 1999; Spruit 2002; Menou et al. 2004; Maeder \& Meynet 2004; Eggenberger et al. 2005; Braithwaite \& Spruit 2005; Braithwaite 2006; Brun \& Zahn 2006). Finally, waves constitute the last transport process in single stars where they are also interacting with rotation. Internal waves, which are excited at the borders with convective zones, propagate inside radiation zones where they extract or deposit angular momentum where they are damped leading to a modification of the angular velocity profile and thus of the chemicals distribution (cf., Goldreich \& Nicholson 1989; Talon et al. 2002 ; Talon \& Charbonnel 2003, 2004, 2005; Rogers \& Glatzmaier 2005). Note also that rotation modifies stellar winds and mass losses (cf., Maeder 1999).

On the other hand, in closed binary systems, where the companion could be a star as well as a planet, there are transfers of angular momentum between the star, its companion and the orbit due to the dissipation acting on flows induced by the tidal potential; that could be the equilibrium tide (cf., Zahn 1966) due to the hydrostatic adjustement of the star or the dynamical tide which is due to the tidal excitation of internal waves (cf., Zahn 1975. This dynamical evolution modifies the internal rotation of each component that have consequences on the properties of their internal transport.

To conclude, all the processes, with which rotation interacts, transport angular momentum and matter that modifies the internal angular velocity, the chemical composition and the nucleosynthesis. Therefore, differential rotation has imperatively to be taken into account to get a coherent picture of the internal dynamics and the evolution of the stars. To achieve this goal, advances in the secular magnetohydrodynamics of stellar radiation zones have been made.

First, rotational transport of type I - where the angular momentum and the chemical species are transported by the meridional circulation and by the hydrodynamical turbulence due to shear instabilities - has been studied. Its present modelling has been generalized to treat simultaneously the bulk of radiation zones and their interfaces with convective zones, the tachoclines (cf., Mathis \& Zahn 2004). Next, a new prescription for the horizontal turbulent transport has been obtained. It has been derived from CouetteTaylor laboratory experiments that enable the study turbulence in differentially rotating flows (cf., Mathis et al. 2004). However, the introduction of these two hydrodynamical mechanisms in stellar models leads to results which fail to reproduce the observations of solar-type stars, because these have been slowed down by the wind during their evolution and hence the rotational processes are less efficient. Therefore, rotational transport of type II has been introduced. The chemical species are still transported by meridional circulation and turbulence, but angular momentum is carried by an another process: the two candidates being the magnetic field and internal waves. First, the effects of a fossil magnetic field have been introduced in a consistent way. The action of turbulence, 


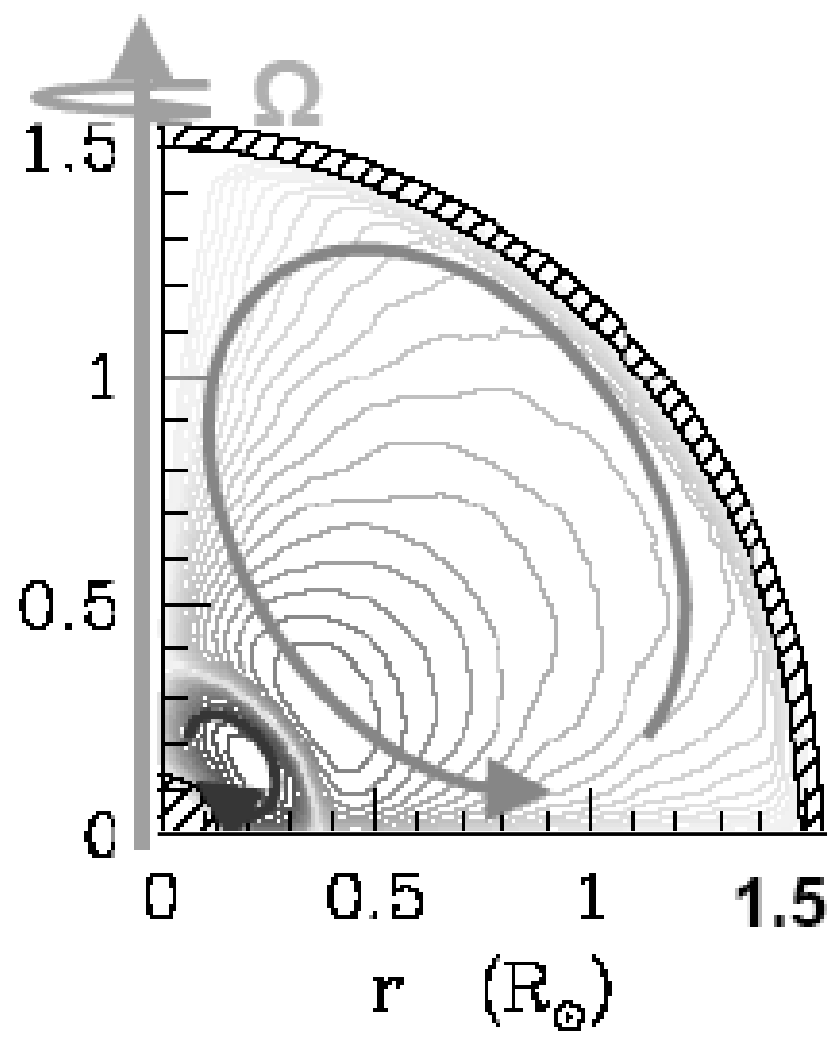

Figure 8. Meridional circulation currents in a $1.5 \mathrm{M}_{\odot}$ star with a solar metallicity and $v_{\text {ini }}=100 \mathrm{~km} \mathrm{~s}^{-1}$. The age is $7.604 \times 10^{8}$ years with a central hydrogen mass fraction $X_{c}=0.57$. In this model, the outer cell is turning counterclockwise allowing the equatorial extraction of angular momentum by the wind.

differential rotation, and meridional circulation on the field, and also its feed-back on momentum and heat transport, are taken into account (cf., Mathis \& Zahn 2005). Next, the effects of the Coriolis force have been introduced in the modelling of internal waves (cf., Mathis \& Zahn 2005). This allows the inclusion of gravito-inertial waves in the description of the angular momentum transport. Finally, a coherent treatment of tidal processes has been derived.

On the other hand, numerical simulations, based on those theoretical results which allow the following in $2 \mathrm{D}$ of the secular hydrodynamics of rotating stars, have been developed with the associated diagnosis tools to identify the dominant processes in angular momentum transport, meridional circulation, and chemical species mixing. The first step - where we assume that the anisotropic turbulence in stellar radiation zones enforces a shellular rotation law $\bar{\Omega}(r)$ - has been achieved. Work is now in progress to implement in stellar evolution codes the differential rotation in latitude and the transport by the magnetic field and the gravito-inertial waves, those two last processes being crucial to understanding the internal angular momentum transport in the Sun and the properties of low-mass stars. Moreover, theoretical work is now underway on the description of MHD instabilities and of the internal-wave excitation to improve our description of stellar radiation zones in support of helioseismology and of asteroseismology. 


\subsection{A new era of asteroseismology: hot stars}

The theoretical $g$-mode frequency spectrum is by far the most dense and their periods are the longest, making $g$-mode pulsators more difficult to study than $p$-mode pulsators. During the last few years, big steps forward have been made for the 'hot' stars, i.e., pulsating stars situated along the main sequence which are hotter than solar type stars.

The $\beta$ Cep and $\delta$ Sct stars are early-B and mid-A to early-F type stars, respectively, pulsating in low order $p$ - and $g$-modes with periods of the order of hours. The modes are excited by the $\kappa$ mechanism acting on a partial ionization zone of metals (Fe) and helium (He), respectively. Currently, more than $100 \beta$ Cep and $1200 \delta$ Sct stars are known. The multi-periodic ones have beat-periods of weeks up to months. Recently, a magnetic field of a few hundred Gauss has been detected for three $\beta$ Cep stars (Henrichs et al. 2000; Neiner et al 2003b; Hubrig et al 2006). The best studied $\beta$ Cep star is $\nu$ Eri (HD 29248), for which large scale multi-site campaigns have been organized in 2002 - 2003 (Handler et al. 2004; Aerts et al 2004; De Ridder et al. 2004) and 2003-2004 (Jerzykiewicz et al. 2005). A total of 14 independent frequencies are found, including the radial fundamental $(\ell=0$, $\left.p_{1}\right)$, three rotationally-split triplets $\left(\ell=1, g_{1}, p_{1}\right.$, and $\left.p_{2}\right)$, and two unidentified highorder $g$-modes. Asteroseismic modeling of $\beta$ Cep stars based on a few identified modes (including the radial fundamental or first overtone) has led to evidence for convective core overshoot and in two cases for non-rigid rotation (the core rotates faster than the surface layers). For the observed modes of $\nu$ Eri to be unstable in the theoretical models, a metal enhancement in the whole interior (Ausseloos et al. 2004) or in the driving region only (Pamyatnykh et al. 2004) has been assumed.

Currently, FG Virginis is the best studied $\delta$ Sct star, for which multi-site campaigns have been organized within the Delta Scuti Network between 1992 and 2004. So far, 67 independent frequencies have been detected in the photometric data, which confirms the theoretical prediction of a large number of unstable modes (Breger et al. 2005). This includes a lot of high frequencies with an amplitude below $0.2 \mathrm{mmag}$ that cannot be explained with unstable low $\ell$ modes (Daszyńska-Daszkiewicz et al. 2006) and close frequency pairs around the expected positions of radial modes (Breger \& Pamyatnykh 2006). Thirteen of these frequencies have been confirmed in spectroscopic data. The mode identification reveals: $(i)$ a lot of axisymmetric modes, including at least two radial ones; (ii) a rotationally-split $\ell=1$ mode; and (iii) evidence for insufficient convection (Viskum et al. 1998; Breger et al. 1999; Mantegazza \& Poretti 2002; Daszyńska-Daszkiewicz et al. 2005; Zima et al. 2006;). These results are used as input for asteroseismic modeling, which is ongoing at the moment.

The slowly pulsating B (SPB) and $\gamma$ Dor stars are mid-B to late-B and early-F type stars, respectively, pulsating in high-order $g$-modes with periods of the order of days. The modes are excited by the $\kappa$ mechanism acting on the partial ionization zone of metals $(\mathrm{Fe})$ and by a flux blocking mechanism at the base of the convective envelope, respectively. For both classes, more than one hundred (candidate) members are known up to now. The multi-periodic ones have beat-periods of months up to years. Recently, a magnetic field of a few hundred Gauss has been detected for 14 SPB stars (Neiner et al. 2003a; Hubrig et al. 2006). HD 160124 holds the record of the largest number of detected frequencies (8) for an SPB star based on ground-based observations (Waelkens 1991), while 21 frequencies were detected in the 37 days of white light observations obtained with the MOST satellite for the new SPB star HD 163830 (Aerts et al. 2006). The highest frequency is a known artefact of the satellite and the two lowest frequencies could be associated with the star's rotational frequency, but the remaining 18 frequencies can easily be interpreted in 
terms of unstable low-degree $g$-modes. An accurate mode identification is lacking which prevents us from further digging into the seismic models.

For the $\gamma$ Dor stars, we are still in the inventory stage. Large-scale ground-based observation campaigns have been organized both in the northern (Kaye et al. 1999; Henry et al. 2001; Henry \& Fekel 2002; Henry \& Fekel 2003; Fekel et al. 2003; Mathias et al. 2004; Henry \& Fekel 2005; Henry et al. 2005) and southern (Eyer et al. 2002; De Cat et al. 2006) hemisphere to search for members of this class. Large steps forward are achieved by the inclusion of time-dependent convection in the models which enables to calculate the instability of the modes and the theoretical amplitude ratios and phase shifts that can be used for mode identification (Grigahcène et al. 2005; Dupret et al. 2005). The frequency ratio method has led to promising results for $\gamma$ Dor stars. It is based on the first-order asymptotic $g$-mode expression and enables the selection of seismic models that are compatible with the observations as soon as three frequencies are observed (Moya et al. 2005). In case of 9 Aur, a unique model has been found (Moya et al. 2006).

The potential of seismology of 'hot' stars is excellent, and for the hybrid stars it is even better, since both $p$ - and $g$-modes are excited. The currently known (candidate) $\beta$ Cep/SPB hybrids are $\iota$ Her (Chapellier et al. 2000), 53 Psc (Le Contel et al. 2001), $\nu$ Eri (Jerzykiewicz et al. 2005), $\gamma$ Peg (Chapellier et al. 2006), and HD 13745 \& HD 19374 (De Cat et al. 2006), while HD 209295 (Handler et al. 2002) and HD 8801 (Henry \& Fekel $2005)$ are $\delta$ Sct $/ \gamma$ Dor hybrids. For the future, we need: $(i)$ accurate mode identifications, especially for $g$-modes; $(i i)$ the inclusion of magnetic fields, diffusion, and rotation in theoretical models; and (iii) data from multi-site campaigns and/or from satellite missions like MOST, CoRoT, and Kepler.

\section{Future activities}

The future for the fields of helioseismology and asteroseismology is bright. Numerous new initiatives and forthcoming activities and missions were discussed at the meeting. Several of these, including NASA's Solar Dynamics Observatory (expected launch data 2008) and the proposed DynaMICS mission in the field of helioseismology, and CoRoT (launched December 2006) in the area of asteroseismology, have been mentioned already above.

Solar Orbiter, ESA's next mission to study the Sun, is scheduled to be launched in May 2015 according to the current baseline (December 2006). The extended mission will be completed in January 2024. The most interesting aspects of the mission for helioseismology reside in the unique vantage points from which the Sun will be viewed (Gizon 2007 Gizon 2007; Woch \& Gizon 2007). The spacecraft will use multiple gravity-assist manoeuvres at Venus and Earth to reach its science orbit after a cruise phase of about 3.4 years. The orbit design will include two main characteristics, both of which offer novel perspectives for helioseismology. First, Solar Orbiter will make observations away from the ecliptic plane to provide views of the Sun's polar regions. The inclination of the spacecraft's orbit to the Ecliptic will incrementally increase at each Venus swing-by manoeuvre to reach at least $30^{\circ}$ toward the end of the mission. Second, Solar Orbiter will cover a large range of spacecraft-Sun-Earth angles. In combination with data collected from the ground or near-Earth orbit, Solar Orbiter will thus mark the advent of steroscopic helioseismology. One important goal is to gain a better understanding of solar activity and variability by probing the solar interior to higher latitudes and larger depths, beyond what can be achieved with Earth-side observations alone.

The SIAMOIS fourier tachometer (Seismic Interferometer Aiming to Measure Oscillations in the Interior of Stars; <http://siamois.obspm.fr/>) is planned for Dome C in 
Antarctica . The Antarctic site presents the unique advantage of requiring only a single instrument for the continuous observation of a target over several months. This postCoRoT instrument, based on an interferometric method to measure the Doppler shifts induced by the $p$-modes, should be put in operation during the 2011 austral winter with this objective. Two bright stars could be observed simultaneously with two robotic 40-cm telescopes feeding the interferometer through a fiber. There are about ten southern, circumpolar solar-type stars observable, with among them $\alpha$ Cen A and B, with such small telescopes. With future developments on the site, this type of instrument would find its place behind a larger telescope to conduct similar long observations on fainter stars.

The NASA Kepler mission, planned for launch in November 2008, will provide extensive asteroseismic data. Kepler will survey of the order of 100000 stars in a 105-square-degree field in Cygnus, nearly continuously for more than four years. The goal is to detect extrasolar planetary systems through the observation of slight decreases in the luminosity of the stars caused by planetary transits. The bulk of the stars will be observed with a cadence of 30 minutes. However, 512 selected stars will at any given time be observed with a one-minute cadence, hence for example allowing the study of solar-like oscillations. Each such star will be observed for at least three months, and in some cases observations of a given star continuously or repeatedly throughout the mission may be warranted, e.g., to search for frequency variations associated with cyclic activity. For stars of magnitude between 9 and 11 it is estimated that detailed asteroseismic investigations will be possible; for fainter stars simulations indicate that it will be possible to determine the large and small frequency separations, hence providing measures of stellar radii, and evolutionary states. Also, even the longer-cadence data are of great asteroseismic interest for the study of solar-like oscillations in giant stars or, for example, long-period oscillations in $\gamma$ Doradus or slowly pulsating B-type stars.

The CoRoT and Kepler missions, as well as the formerly proposed Eddington mission, highlight the benefits of combining observations for asteroseismology and exo-planets in a single mission. The CoRoT mission will spend five months on each of five fields of 10 square degrees. The asteroseismology component will monitor the light curves of a total of 50 target starst with magnitudes in the range 5 to 9 with a cadence of 32 seconds, yielding a precision on the frequencies of order $0.1 \mu \mathrm{Hz}$. The planet search component will monitor of the order of 10,000 stars in each field with magnitudes in the range 11 to 16; 1000 with a cadence of 32 seconds the rest with a cadence of 512 seconds. The planet search data will also be available for asteroseismology studies.

Looking to the future, PLATO is a new mission concept being developed by a European consortium to be submitted to the European Space Agency for inclusion in the new Cosmic Vision programme. The current concept is to have an aligned cluster of small telescopes with very wide fields of view which will continuously monitor of the order of 100,000 stars for both asteroseismology and planet finding. The concept is currently undergoing industrial studies.

Finally, there are ambitious plans to build SONG (Stellar Oscillations Network Group) (<http://astro.phys.au.dk/SONG〉), which will be a global network of small telescopes equipped with high-resolution spectrographs and dedicated to asteroseismology and planet searches.

\section{References}

Aerts, C., De Cat, P., Handler, G., et al. 2004, MNRAS, 347, 463

Aerts, C., De Cat, P., Kuschnig, R., et al. 2006, ApJ (Letters), 642, L165

Altrock, R., \& Howe, R. 2004, BAAS, 36, 1411 
Andersen, B. 1996, A\& A, 312, 610

Ausseloos, M., Scuflaire, R., Thoul, A., \& Aerts, C. 2004, MNRAS, 355, 352

Balmforth, N. J. 1992, MNRAS, 255, 639

Barban, C., De Ridder, J., Mazumdar, A., et al., 2004, in: D. Danesy (ed.), Proc. SOHO 14/GONG 2004 Workshop, Helio- and Asteroseismology: Towards a Golden Future, ESA SP-559, p. 113

Basu, S., \& Antia, H. M. 2001, MNRAS, 324, 498

Basu, S., \& Antia, H. M. 2003, ApJ, 585, 553

Baudin, F., Samadi, R., Goupil, M.-J., et al. 2005, A\&A A, 433, 349

Beck, J. G., Gizon, L., \& Duvall, T. L. 2002, ApJ (Letters), 575, L47

Bedding, T. R., Kjeldsen, H., Arentoft, T., et al. 2007, ApJ, 663, 1315

Bedding, T. R., Butler, R. P., Carrier, F., et al. 2006, ApJ, 647, 558

Bedding, T. R., Kjeldsen, H., Bouchy, F., et al. 2005, A\&A (Letters), 432, L43

Bedding, T. R., Kjeldsen, H., Butler, R. P., et al. 2004, ApJ, 614, 380

Belkacem, K., Samadi, R., Goupil, M., \& Kupka, F. 2006a, A\&̛A, 460, 173

Belkacem, K., Samadi, R., Goupil, M., Kupka, F., \& Baudin, F. 2006b, A\&̈A, 460, 183

Bouchy, F., Bazot, M., Santos, N. C., Vauclair, S., \& Sosnowska, D. 2005, A\&A, 440, 609

Bouchy, F., \& Carrier, F. 2002, A\&A, 390, 205

Bouchy, F., Maeder, A., Mayor, M., et al. 2004, Nature, 432, 7015

Braithwaite, J. 2006, A\&A, 449, 451

Braithwaite, J., \& Spruit, H. 2005, Nature, 431, 819

Braun, D. C. 1995, ApJ, 451, 859

Breger, M., Lenz, P., Antoci, V., et al. 2005, A\&BA, 435, 955

Breger, M., \& Pamyatnykh, A. A. 2006, MNRAS, 368, 571

Breger, M., Pamyatnykh, A. A., Pikall, H., \& Garrido, R. 1999, A\&GA, 341, 151

Browning, M., Miesch, M. S., Brun, A. S., \& Toomre, J. 2006, ApJ (Letters), 648, 157

Brun, A. S. 2004, Solar Phys., 220, 33

Brun, A. S. 2007, AN 328, 329

Brun, A. S., Miesch, M., \& Toomre, J. 2004, ApJ, 1014, 1073

Brun, A. S., \& Toomre, J. 2002, ApJ, 570, 865

Brun, A. S., \& Zahn, J.-P. 2006, A\& $A$ 457, 665

Busse, F. H. 1982, ApJ, 259, 759

Cally, P. S. 2006, Phil. Trans. Roy. Soc. Lond. A, 364, 333

Cally, P. S., Crouch, A. D., \& Braun, D. C. 2003, MNRAS, 346, 381

Carrier, F., \& Bourban, G. 2003, A\& $A$ (Letters), 406, L23

Carrier, F., Bouchy, F., \& Eggenberger, P. 2003, in: M. J. Thompson, M. S. Cunha \& M. J. P. F.

G. Monteiro (eds.), Asteroseismology Across the HR Diagram (Dordrecht:Kluwer), p. 311

Carrier, F., \& Eggenberger, P. 2006, A\&A, 450, 695

Carrier, F., Eggenberger, P., \& Bouchy, F. 2005a, A\&A, 434, 1085

Carrier, F., Eggenberger, P., D'Alessandro, A., \& Weber, L. 2005b, New Astron., 10, 315

Carrier, F., Kjeldsen, H., Bedding, T. R., et al. 2007, A\&A 470, 1059

Chapellier, E., Le Contel, D., Le Contel, J. M., Mathias, P., et al. 2006, A\&GA, 448, 697

Chapellier, E., Mathias, P., Le Contel, J., et al. 2000, A\&A, 362, 189

Chaplin, W. J., Elsworth, Y., Isaak, G. R., et al. 1998, MNRAS (Letters), 298, L7

Chaplin, W. J., Elsworth, Y., Isaak, G. R., et al. 2003, ApJ (Letters), 582, L115

Chaplin, W. J., Houdek, G., Elsworth, Y., et al. 2005, MNRAS, 360, 859

Charbonneau, P., \& MacGregor, K. B. 1993, ApJ 417, 762

Charbonnel, C., \& Talon, S. 2005, Science, 309, 2189

Chou, D.-Y., \& Dai, D. 2001, ApJ (Letters), 559, L175

Chou, D.-Y., \& Ladenkov, O. 2005, ApJ, 630, 1206

Claudi, R. U., Bonanno, A., Leccia, S., et al. 2005, A\&A (Letters), 429, L17

Clune, T. L., Elliott, J. R., Glatzmaier, G. A., et al. 1999, Parallel Comput. 25, 361

Couvidat, S., Turck-Chièze, S., \& Kosovichev, A. 2003a, ApJ, 599, 1434

Couvidat, S., Garcìa, R. A., Turck-Chièze, S., et al. 2003b, ApJ (Letters), 597, L77 
Crouch, A. D., \& Cally, P. S. 2003, Solar Phys. 214, 201

Crouch, A. D., \& Cally, P. S. 2005, Solar Phys. 227, 1

Crouch, A. D., Cally, P. S., Charbonneau, P., et al. 2005, MNRAS, 363, 1188

Daszyńska-Daszkiewicz, J., Dziembowski, W. A., \& Pamyatnykh, A. A. 2006, Mem. S.A.It., 77, 113

Daszyńska-Daszkiewicz, J., Dziembowski, W. A., Pamyatnykh, A. A., et al. 2005, A\& A, 438, 653

De Cat, P., Eyer, L., Cuypers, J., et al. 2006, A\&A, 449, 281

De Ridder, J., Barban, C., Carrier, F., et al. 2006, A\&A, 448, 689

De Ridder, J., Telting, J. H., Balona, L. A., et al. 2004, MNRAS, 351, 324

Dikpati, M., \& Charbonneau, P. 1999, ApJ, 518, 508

Dikpati, M., \& Gilman, P. A. 2001, ApJ, 559, 428

Dikpati, M., Cally, P. S., \& Gilman, P. A. 2004, ApJ, 610, 597

Dupret, M.-A., Grigahcène, A., Garrido, R., Gabriel, M., \& Scuflaire, R. 2005, A $\& A, 435,927$

Eggenberger, P., Maeder, A., \& Meynet, G. 2005, A\& A (Letters), 440, L9

Eyer, L., Aerts, C., van Loon, M., Bouckaert, F., \& Cuypers, J. 2002, in: C. Sterken \& D. W. Kurtz (eds.), Observational Aspects of Pulsating B-and A Stars, ASP-CS 256, 203

Fekel, F. C., Warner, P. B. \& Kaye, A. B. 2003, AJ, 125, 2196

Fletcher, S. T., Chaplin, W. J., Elsworth, Y., Schou, J., \& Buzasi, D. 2006, MNRAS, 371, 935

Frandsen, S., Carrier, F., Aerts, C., et al. 2002, A\&A (Letters), 394, L5

Garaud, P. 2001, MNRAS 324, 68

Garaud, P. 2002, MNRAS, 329, 1

Garaud, P. 2002, MNRAS, 335, 707

García, R., Corbard, T., Chaplin, W. J., et al. 2004, Solar Phys., 220, 269

García, R., Turck-Chize, S., Boumier, P., et al. 2005, A\&\&A, 442, 385

García, R., Turck-Chièze, S., Jiménez-Reyes, S. J., et al. 2006, in: K. Fletcher \& M. J. Thompson (eds.), SOHO 18/ GONG 2006/ HELAS I, Beyond the Spherical Sun: Towards a Golden Future, ESA SP-624, 23.1

Giles, P. M. 1999, PhD thesis, Stanford University, USA

Giles, P. M., Duvall, T. L., Scherrer, P. H., \& Bogart, R. S. 1997, Narture, 390, 52

Gizon, L. 2004, Solar Phys., 224, 217

Gizon, L. 2007, in: The Second Solar Orbiter Workshop, ESA SP-641, in press

Gizon, L., \& Rempel, M. 2006, in: K. Fletcher \& M. J. Thompson, (eds.), SOHO 18/ GONG 2006/ HELAS I, Beyond the Spherical Sun: Towards a Golden Future, ESA SP-624, 129.1

Goldreich, P., \& Keeley, D. A. 1977, ApJ, 212, 243

Goldreich, P., \& Nicholson, P. D. 1989, ApJ, 342, 1079

González Hernández, I., Komm, R., Hill, F., et al. 2006, ApJ, 638, 576

Grigahcène, A., Dupret, M.-A., Gabriel, M., Garrido, R., \& Scuflaire, R. 2005, A\&A, 434, 1055

Gryanik, V., \& Hartmann, J. 2002, J. Atmos. Sci., 59, 2729

Guenther, D. B., Kallinger, T., Reegen, P., et al. 2005, ApJ 635, 547

Handler, G., Balona, L. A., Shobbrook, R. R., et al. 2002, MNRAS, 333, 262

Handler, G., Shobbrook, R. R., Jerzykiewicz, M., et al. 2004, MNRAS, 347, 454

Henrichs, H. F., de Jong, J. A., Donati, D.-F., et al. 2000, in: Yu.V. Glagolevskij \& I. I. Romanyuk (eds.), Magnetic Fields of Chemically Peculiar and Related Stars, Proc. Intern. Meeting Special Astrophysical Observatory, September 23-27, 1999, p.57

Henry, G. W., \& Fekel, F. C. 2002, PASP, 114, 988

Henry, G. W., \& Fekel, F. C. 2003, AJ 126, 3058

Henry, G. W., \& Fekel, F. C. 2005, AJ, 129, 2026

Henry, G. W., Fekel, F. C., \& Henry, S. M. 2005, AJ, 129, 2815

Henry, G. W., Fekel, F. C., Kaye, A. B. \& Kaul, A. 2001, AJ, 122, 3383

Hill, H., Frohlich, C., Gabriel, M. \& Kotov, V. A. 1991, in: A. N. Cox, W. C. Livingston \& M. S. Matthews (eds.), Solar Interior and Atmosphere (Tucson: Univ. of Arizona Press), p. 562

Houdek, G. 2006, in: K. Fletcher \& M. J. Thompson, (eds.), SOHO 18/ GONG 2006/ HELAS I, Beyond the Spherical Sun: Towards a Golden Future, ESA SP-624, p. 28.1

Howe, R. 2003, in: H. Sawaya-Lacoste (ed.), Proceedings of SOHO 12 / GONG+ 2002. Local and Global Helioseismology: the Present and Future, ESA SP-517, p. 81 
Howe, R., Christensen-Dalsgaard, J., Hill, F., et al. 2000, Science, 287, 2456

Howe, R., Christensen-Dalsgaard, J., Hill, F. 2005, ApJ, 634, 1405

Howe, R., González Hernández, I., Hill, F. \& Komm, R. 2006a, in: K. Fletcher (ed.), SOHO 18/GONG 2006/HELAS I, Beyond the Spherical Sun. A New Era of Helio- and Asteroseismology, ESA SP-624, 68.1

Howe, R., Komm, R., \& Hill, F. 2002, ApJ, 580, 1172

Howe, R., Hill, F., Komm, R., et al. 2006b, paper presented at HMI Team meeting, Monterey, California, February 2006

Howe, R., Komm, R., Hill, F., et al. 2006c, Solar Phys., 235, 1

Hubrig, S., Briquet, M., Schöller, M., et al. 2006, MNRAS (Letters), 369, L61

Jerzykiewicz, M., Handler, G., Shobbrook, R. R., et al. 2005, MNRAS, 360, 619

Jouve, L., \& Brun, A. S. 2007, A\&\&A, 474, 239

Kaye, A. B., Henry, G. W., Fekel, F. C., et al. 1999, AJ 118, 2997

Kitchatinov, L. L., \& Rüdiger, G. 1993, A\& A, 276, 96

Kjeldsen, H., Bedding, T. R., Baldry, I. K., et al. 2003, AJ, 126, 1483

Kjeldsen, H., Bedding, T. R., Butler, R. P., et al. 2005, ApJ, 635, 1281

Komm, R. W. 1994, Solar Phys., 149, 417

Komm, R. W., Howard, R. F., \& Harvey, J. W. 1993, Solar Phys., 147, 207

Komm, R. W., Howe, R., \& Hill, F. 2000, ApJ, 543, 472

Komm, R. W., Howe, R., \& Hill, F. 2002, ApJ, 572, 663

Komm, R. W., Howe, R., \& Hill, F. 2006, Adv. Space Res., 38, 845

Korzennik, S. G., Rabello-Soares, M. C., \& Schou, J. 2004, ApJ, 602, 481

Kumar, P., Quataert, E. J. \& Bahcall, J. N. 1996, ApJ (Letters), 458, L83

Le Contel, J.-M., Mathias, P., Chapellier, E., \& Valtier, J.-C. 2001, A\&\&A, 380, 277

Lefebvre, S., \& Kosovichev, A. G. 2005, ApJ (Letters), 633, L149

Lefebvre, S., Kosovichev, A. G., Nghiem, P., et al., 2006, in: K. Fletcher \& M. J. Thompson (eds.), SOHO 18/ GONG 2006/ HELAS I, Beyond the Spherical Sun: Towards a Golden Future, ESA SP-624, 9.1

Libbrecht, K. G., \& Woodard, M. F. 1990, Nature, 345, 779

Maeder, A. 1999, A\&A, 347, 185

Maeder, A. 2003, A\&BA, 399, 263

Maeder, A., \& Meynet, G. 2004, A\&SA, 422, 225

Maeder, A., \& Zahn, J.-P. 1998, A\&A, 334, 1000

Mantegazza, L., \& Poretti, E. 2002, A\& A, 396, 911

Martić, M., Lebrun, J.-C., Appourchaux, T., \& Korzennik, S. G. 2004a, A\&A A, 418, 295

Martić, M., Lebrun, J. C., Appourchaux, T., \& Schmitt, J. 2004b, in: D. Danesy (ed.), Proc. SOHO 14/GONG 2004 Workshop, Helio- and Asteroseismology: Towards a Golden Future, ESA SP-559, p. 563

Mathias, P., Le Contel, J.-M., Chapellier, E., et al. 2004, A\&A, 417, 189

Mathis, S., Palacios, A., \& Zahn, J.-P. 2004, A\&GA, 425, 243

Mathis, S., \& Zahn, J.-P. 2004, A\&A, 425, 229

Mathis, S., \& Zahn, J.-P. 2005, A\&A, 440, 653

Mathis, S., \& Zahn, J.-P. 2005, in: F. Casoli, T. Contini, J. M. Hameury, \& L. Pagani (eds.) Proc. Semaine de l'Astrophysique Francaise 2A-2005, EdP-Sciences CS, 319

Mathur, S.,Turck-Chièze, S., Couvidat, S., García, R. 2006, in: K. Fletcher \& M. J. Thompson, (eds.), SOHO 18/ GONG 2006/ HELAS I, Beyond the Spherical Sun: Towards a Golden Future, ESA SP-624, p. 95.1

Matthews, J. M., Kuschnig, R., Guenther, D. B., et al. Nature, 430, 51. Erratum: 430, 921

Menou, K., Balbus, S. A., Spruit, H. C. 2004, ApJ, 607, 564

Miesch, M. S., Brun, A. S., \& Toomre, J. 2006, ApJ, 641, 618

Miesch, M. S., Elliott, J. R., Toomre, J., et al. 2000, ApJ, 532, 593

Mosser, B., Bouchy, F., Catala, C., et al. 2005, A $\mathscr{E} A$ (Letters), 431, L13

Moya, A., Grigahcene, A., Suárez, J. C., et al. 2006, Mem. S.A.It., 77, 466

Moya, A., Suárez, J. C., Amado, P. J., Martin-Ruíz, S., \& Garrido, R. 2005, A\&A, 432, 189

Neiner, C., Geers, V. C., Henrichs, H. F., et al. 2003a, A\&SA, 406, 1019

Neiner, C., Henrichs, H. F., Floquet, M., et al. 2003b, A\&A A, 411, 565 
Pallé, P. L. 1991, Adv. Space Res., 11 (4), 29

Pamyatnykh, A. A., Handler, G., \& Dziembowski, W. A. 2004, MNRAS, 350, 1022

Parker, E. N. 1993, ApJ, 408, 707

Rabello-Soares, M. C., Basu, S., Christensen-Dalsgaard, J., et al. 2000, Sol. Phys., 193, 345

Rabello-Soares, M. C. Korzennik, S. G., \& Schou, J. 2007, Adv. Space Res., E22-0005-06, in press

Rempel, M. 2005, ApJ, 622, 1320

Rempel, M. 2006, ApJ, 647, 662

Rieutord, M. 2006, in: M. Rieutord \& B. Dubrulle (eds.), Stellar Fluid Dynamics and Numerical Simulations: From the Sun to Neutron Stars, EAS-PS, 21, 275

Rieutord, M., \& Zahn, J.-P. 1995, A\&A, 296, 127

Rogers, T., \& Glatzmaier, G. 2005, MNRAS, 364, 1135

Salabert, D., \& Jiménez-Reyes, S. J. 2006, in: K. Fletcher \& M. J. Thompson, (eds.), SOHO 18/ GONG 2006/ HELAS I, Beyond the Spherical Sun: Towards a Golden Future, ESA SP-624, 90.1

Salabert, D., Chaplin, W. J., Elsworth, Y., New, R., \& Verner, G. A. 2007, A\&AA, 463, 1181

Samadi, R., Belkacem, K., Goupil, et al. 2007, in: F. Kupka, I. W. Roxburgh \& K. L. Chan (eds.) Convection in Astrophysics, Proc. IAU Symp. No. 239 (Cambridge: CUPP), p. 119

Samadi, R., \& Goupil, M. 2001, A\&A 370, 136

Samadi, R., Nordlund, A, Stein, R. F., Goupil, M. J., \& Roxburgh, I. 2003a, A\&̛A 404, 1129

Schou, J., \& Buzasi, D. L. 2001, in: A. Wilson \& P. L. Pallé (eds.), SOHO 10/GONG 2000: Helio- and Asteroseismology at the Dawn of the Millennium, ESA SP-464, 391

Schunker, H., Braun, D. C., Cally, P. S., \& Lindsey, C. 2005, ApJ (Letters), 621, L149

Schunker, H., \& Cally, P. S. 2006, MNRAS, 372, 551

Spruit, H. C. 1999, A\& A 349, 189

Spruit, H. C. 1991, in: J. Toomre \& D. O.Gough (eds), Challenges to Theories of the Structure of Moderate Mass Stars, Lecture Notes in Physics, 388, 121

Spruit, H. C. 2002, A\&A, 381, 923

Spruit, H. C. 2003, Solar Phys., 213, 1

Stello, D., Kjeldsen, H., Bedding, T. R. \& Buzasi, D. 2006, A\&̈A, 448, 709

Talon, S., \& Charbonnel C. 2003, A\&A, 405, 1025

Talon, S., \& Charbonnel C. 2004, A\&A, 418, 1051

Talon, S., \& Charbonnel C. 2005, A\&A A, 440, 981

Talon, S., Kumar P., \& Zahn J.-P. 2002, ApJ (Letters) 574, L175

Talon, S., \& Zahn, J.-P. 1997, A\&̈A, 317, 749

Tripathy, S. C., Hill, F., Jain, K., \& Leibacher, J. W. 2007, Solar Phys., submitted

Turck-Chièze, S., Couvidat, S., Kosovichev, A. G., et al. 2001, ApJ (Letters), 555, L69

Turck-Chièze, S., García, R. A., Couvidat, S., et al. 2004, ApJ, 604, 455

Turck-Chièze, S., Appourchaux, T., \& Ballot, J. 2005, in: F. Favata, J. Sanz-Forcada, A. Giménez \& B. Battrick (eds.), Trends in Space Science and Cosmic Vision 2020, Proc. 39th ESLAB Symposium, ESA SP-588, 193

Turck-Chièze, S. 2006a, Adv. Space Res., 37, 1569

Turck-Chièze, S., Carton, P.-H., Ballot, J., et al. 2006b, Adv. Space Res., 38, 1812

Turck-Chièze, S., et al. 2006c, in: K. Fletcher \& M. J. Thompson, (eds.), SOHO 18/ GONG 2006/ HELAS I, Beyond the Spherical Sun: Towards a Golden Future, ESA SP-624, 24.1

Viskum, M., Kjeldsen, H., Bedding, T. R., et al. 1998, A\&A, 335, 549

Vorontsov, S. V., Christensen-Dalsgaard, J., Schou, J., et al. 2002, Science, 296, 101

Waelkens, C. 1991, A\&\&A, 246, 453

Woch, J., \& Gizon, L. 2007, AN, 328, 362

Woodard, M. F., \& Noyes, R. W. 1985, Nature, 318, 445

Zahn, J.-P. 1966, Ann. d'Astrophys. 29, 313 (I), 489 (II)

Zahn, J.-P. 1975, A\&\&A, 41, 329

Zahn, J.-P. 1992, A\&A, 265, 115

Zhao, J., \& Kosovichev, A. G. 2004, ApJ, 603, 776

Zima, W., Wright, D., Bentley, J., et al. 2006, A\&A, 455, 235 